\title{
Statistical analysis of C/NOFS planar Langmuir probe data
}

\author{
E. Costa ${ }^{1}$, P. A. Roddy ${ }^{2}$, and J. O. Ballenthin ${ }^{2}$ \\ ${ }^{1}$ Centro de Estudos em Telecomunicações (CETUC) Pontifícia Universidade Católica do Rio de Janeiro (PUC-Rio) Rua \\ Marquês de São Vicente 225 Gávea, 22451-900 Rio de Janeiro, RJ, Brasil \\ ${ }^{2}$ Space Vehicles Directorate, Air Force Research Laboratory Kirtland Air Force Base, NM, USA
}

Correspondence to: E. Costa (epoc@ cetuc.puc-rio.br) and P. A. Roddy (patrick.roddy@us.af.mil)

Received: 10 October 2013 - Revised: 24 March 2014 - Accepted: 25 May 2014 - Published: 14 July 2014

\begin{abstract}
The planar Langmuir probe (PLP) onboard the Communication/Navigation Outage Forecasting System (C/NOFS) satellite has been monitoring ionospheric plasma densities and their irregularities with high resolution almost seamlessly since May 2008. Considering the recent changes in status of the C/NOFS mission, it may be interesting to summarize some statistical results from these measurements. PLP data from 2 different years (1 October 2008-30 September 2009 and 1 January 2012-31 December 2012) were selected for analysis. The first data set corresponds to solar minimum conditions and the second one is as close to solar maximum conditions of solar cycle 24 as possible at the time of the analysis. The results from the analysis show how the values of the standard deviation of the ion density which are greater than specified thresholds are statistically distributed as functions of several combinations of the following geophysical parameters: (i) solar activity, (ii) altitude range, (iii) longitude sector, (iv) local time interval, (v) geomagnetic latitude interval, and (vi) season.
\end{abstract}

Keywords. Ionosphere (ionospheric irregularities)

\section{Introduction}

The Communication/Navigation Outage Forecasting System (C/NOFS) satellite was launched on 16 April 2008 into a low inclination $\left(13^{\circ}\right)$, elliptical orbit (perigee and apogee approximately equal to 400 and $850 \mathrm{~km}$, respectively). Data from a suite of sensors on board the satellite drive an equatorial ionospheric model to forecast the onset of plasma instability, as well as its evolution into plasma bubbles (Retterer, 2010). Due to its orbital period, these sensors are able to revisit neighbor regions in space at $90 \mathrm{~min}$ intervals, measuring different ionospheric parameters with high resolution. The list of C/NOFS science objectives has been organized into three categories to (1) understand physical processes active in the background ionosphere and thermosphere in which plasma instabilities grow, (2) identify mechanisms that trigger or quench the plasma irregularities, and (3) determine how the plasma irregularities affect the propagation of electromagnetic waves (de La Beaujardière et al., 2004).

Interesting new observations which may be associated with the solar-minimum conditions that existed during the first months of operation were reported very soon after the launch of the $\mathrm{C} / \mathrm{NOFS}$ satellite. The detailed Introduction by Nishioka et al. (2011) summarized these findings, with direct links to the original references. Next, they discussed apparently contradictory observations of plasma density irregularities and VHF scintillation activity at Ancon, Peru, with the additional support of simultaneous data from a groundbased GPS network, a digisonde, and the Jicamarca incoherent scatter radar. In continuation of the initial studies related to deep solar-minimum conditions, Huang et al. (2011) investigated the generation and evolution of plasma bubbles and broad plasma depletions based on three different evenings of C/NOFS observations. They proposed a new mechanism through which a series of plasma bubbles are created in the evening sector by the Rayleigh-Taylor instability. The longitudinal widths of the plasma bubbles continuously grow, in such a way that multiple bubbles with regular sizes can merge to form wider bubbles or a broad depletion. The plasma bubbles are quenched through their fillings with newly produced particles generated by photoionization processes near dawn.

Additionally, results from electric field measurements by the $\mathrm{C} / \mathrm{NOFS}$ vector electric field instrument (VEFI) were applied to the assimilative physics-based ionospheric model (PBMOD) (Su et al., 2009, 2011) to reproduce postmidnight 
and dawn observations of large-scale density depletions in the Northern Hemisphere summer during solar-minimum conditions.

The above paragraphs illustrate the important role of one particular C/NOFS instrument, its onboard planar Langmuir probe (PLP), which has been monitoring ionospheric plasma densities and their irregularities with high resolution (at $512 \mathrm{~Hz}$ during satellite eclipses) almost seamlessly since May 2008. Considering the recent changes in status of the $\mathrm{C} / \mathrm{NOFS}$ mission, it may be interesting to summarize some statistical results from the PLP measurements. This is the objective of the present contribution. Therefore, it complements previous work by Roddy et al. (2010) and Dao et al. (2011), which also analyzed data corresponding to low solar activity conditions. The first authors processed $\Delta N_{\mathrm{i}} / N_{\mathrm{i}}$ records, calculated from the standard deviation $\Delta N_{\mathrm{i}}$ of ion density fluctuations around its $1 \mathrm{~s}$ average $N_{\mathrm{i}}$, to provide statistics of the ability of the C/NOFS PLP to detect scintillation observed by the Scintillation Network Decision Aid (SCINDA) network (Groves et al., 1997) at Christmas Island ( $\left.3^{\circ} \mathrm{N}, 157^{\circ} \mathrm{W}\right)$. The second authors defined the ambient density as an envelope $N_{\mathrm{i} \text {, env }}$ connecting the local maxima of ion density measurements evaluated every $60 \mathrm{~s}$ (corresponding to approximately $450 \mathrm{~km}$ in space) with the help of spline interpolation. Next, they subtracted the measurements from the envelope to obtain the deviation $\delta N_{\mathrm{i}}$ from the ambient density and computed the normalized density depletion $\delta N_{\mathrm{i}} / N_{\mathrm{i}, \text { env }}$. Finally, they analyzed the average $\delta N_{\mathrm{i}} / N_{\mathrm{i} \text {,env }}$ in $1 \mathrm{~h}$ local time (LT) bins.

The C/NOFS program was not the first to provide data for similar studies. Some of the previous programs will be briefly reviewed in the following and the results of particular interest to the present contribution will be listed.

Kil and Heelis (1998) used data obtained from the retarding potential analyzer (RPA) onboard the Atmosphere Explorer-E (AE-E) satellite, kept with an inclination approximately equal to $19.7^{\circ}$. They analyzed data taken during essentially circular orbits in the period from 1977 to 1981 , divided into two altitude regions: (i) below $300 \mathrm{~km}$ in 1977; or (ii) from 350 to $450 \mathrm{~km}$ in the other years of the period. At these altitudes, the satellite velocity was approximately equal to $8 \mathrm{~km} \mathrm{~s}^{-1}$. The yearly-averaged absolute $10.7 \mathrm{~cm}$ solar flux density values were $78,129,173,179$, and $182 \mathrm{sfu}$ (solar flux unit) in these five years, respectively. Note that 1 sfu corresponds to $10^{-22} \mathrm{~W} \mathrm{~m}^{-2} \mathrm{~Hz}^{-1}$. The RPA onboard AE-E operated over repetitive $8 \mathrm{~s}$ cycles. During each cycle, approximately $3 \mathrm{~s}$ were used to measure a signal that was proportional to the total ion concentration. The signal was sampled at $112 \mathrm{~Hz}$, providing a resolution of stationary structures with scale sizes between approximately $75 \mathrm{~m}$ and $25 \mathrm{~km}$. The intensity of the structure over this period $(3 \mathrm{~s}$ or, equivalently, $25 \mathrm{~km}$ ) was measured as the percentual rms (root mean square) deviation $\sigma_{\mathrm{KH}}$ of the signal from a straight line. The following distributions were presented and discussed by Kil and Heelis (1998): (i) occurrence probability of structures with $\sigma_{\mathrm{KH}}>1 \%$ and $\sigma_{\mathrm{KH}}>5 \%$ as functions of local time (30 min bins); (ii) occurrence probability of irregularities with $\sigma_{\mathrm{KH}}>1 \%$ and average intensity of irregularities as functions of season (3-month bins), longitude sector ( $30^{\circ}$ bins), and local time interval ( $2 \mathrm{~h}$ bins); (iii) occurrence probability of structures with $\sigma_{\mathrm{KH}}>1 \%$ as functions of season (3-month bins) and magnetic latitude ( $30^{\circ}$ bins); and (iv) occurrence probability of irregularities with $\sigma_{\mathrm{KH}}>1 \%$ as functions of season (three-month bins) and longitude sector $\left(30^{\circ}\right.$ bins). All these distributions resulted from data recorded in the altitude interval from 350 to $450 \mathrm{~km}$.

Huang et al. (2002) and Burke et al. (2004a) examined more than 75000 latitudinal ion density profiles measured from 19:30 to 21:30 LT by instruments onboard Defense Meteorological Satellite Program (DMSP) satellites between 1989 and 2001. The extremes of this interval approximately coincide with the maxima of the intense solar cycles 22 and 23 , when their early-averaged absolute $10.7 \mathrm{~cm}$ solar flux density reached the values 190 and $165 \mathrm{sfu}$, respectively. All DMSP satellites follow Sun-synchronous circular orbits. The common inclination, altitude, and period of the orbits are equal to $98.7^{\circ}, 840 \mathrm{~km}$, and $102 \mathrm{~min}$, respectively. The corresponding satellite velocity is close to $7.5 \mathrm{~km} \mathrm{~s}^{-1}$. Each satellite completes 14 orbits per day, with a precession rate of $25^{\circ}$ per orbit. The sections of the above profiles recorded within $\pm 30^{\circ}$ from the magnetic equator were searched for equatorial plasma bubbles (EPBs), characterized by decreases and recoveries of the ion density by factors of two or greater in relation to the corresponding ambient values. No distinction was made between one or several of such plasma density depletions per orbit. Thus, this study was concentrated on intermediate- to large-scale (tens to hundreds of kilometers) irregularities. Slightly over 8300 EPBs were identified. The occurrence rates of EPBs were determined from the set of ratios between the number of orbits with EPBs and total number of orbits in each bin. Huang et al. (2002) classified the results into $24 \times 4$ (longitude sector, season) bins to determine seasonal and solar-cycle variability of the EPB occurrence rates. They also processed the ion densities measured along 200 orbits with no EPB activity in the evening sector during 1991 and 1996. They found that the plasma density decreased from $(3.6 \pm 2.2) \times 10^{5} \mathrm{~cm}^{-3}$ to $(1.08 \pm 0.41) \times 10^{4} \mathrm{~cm}^{-3}$ between solar maximum and minimum. Burke et al. (2004a) assumed $24 \times 12$ (longitude sector, 1-month) bins, each containing approximately 250 samples, to investigate the effects from the strength of the equatorial magnetic field and its angle with the dusk terminator line on the same rate. Burke et al. (2004b) updated their analysis to include similar data recorded during solar maximum 2002.

The ion trap sensor onboard the scientific satellite of the Republic of China (ROCSAT-1) observed irregularities in the topside ionospheric ion density along $35^{\circ}$ inclination, $600 \mathrm{~km}$ circular orbits. At this altitude, the satellite velocity was approximately equal to $7.6 \mathrm{~km} \mathrm{~s}^{-1}$. Su et al. (2006) used the $1 \mathrm{~s}$ averaged values of these measurements from March 1999 
to June 2004 in their studies. The yearly-averaged absolute $10.7 \mathrm{~cm}$ solar flux density values were 138, 162, 165, 162, 117 , and $96 \mathrm{sfu}$ in these 6 years, respectively. Initially, ten consecutive average values $n_{\mathrm{i}}(1 \leq i \leq 10)$ were linearly detrended and the fitted values $n_{\mathrm{oi}}$ at the corresponding data points were obtained. The intensity of the irregularities in the $10 \mathrm{~s}$ data segment was characterized by the ratio $\sigma_{\mathrm{SU}}$ between the square root of the average value of $\left[\log \left(n_{\mathrm{i}} / n_{\mathrm{oi}}\right)\right]^{2}$ and the average value of $\log \left(n_{\mathrm{oi}}\right)$. This intensity measure is affected by irregularities with scale sizes between 7.5 and $75.0 \mathrm{~km}$. The ratio between the time duration when $\sigma_{\mathrm{SU}}>0.3 \%$ and the satellite transit time determined the occurrence probability in each $1^{\circ} \times 1^{\circ}$ square grid in longitude and latitude. The auxiliary parameters local time, season, Kp index, and daily solar flux intensity were also recorded for studies of density irregularity occurrence conditions. Su et al. (2006) presented and discussed the following distributions of the occurrence probability of structures with $\sigma_{\mathrm{SU}}>0.3 \%$ as functions of (i) longitude and latitude $\left(1^{\circ} \times 1^{\circ}\right.$ square bins $)$, for each season; (ii) season (3-month bins) and longitude sector $\left(10^{\circ}\right.$ bins); (iii) season ( 3 -month bins) and dip latitude ( $5^{\circ}$ bins); and (iv) season (3-month bins) and local time ( $1 \mathrm{~h}$ bins), for $\mathrm{Kp}<3$. They claim that, had a linear scale been used to estimate $\sigma_{\mathrm{SU}}$, the adopted threshold would be roughly equivalent to the one used by Kil and Heelis (1998). The set of distributions described in (ii) were compared with corresponding ones obtained by McClure et al. (1998) using AE-E data. These authors characterized the intensity of irregularities by examining the first $3 \mathrm{~s}$ of RPA data of eight consecutive $8 \mathrm{~s}$ cycles. A $64 \mathrm{~s}$ irregularity patch (equivalent to $500 \mathrm{~km}$ ) was only considered in the analysis when at least three (out of eight) values of $\sigma_{\mathrm{KH}}$ and the average ion density exceeded $0.5 \%$ and $5 \times 10^{3} \mathrm{~cm}^{-3}$, respectively.

The success of the C/NOFS program is partially measured by the already vast but still increasing related literature. A more thorough review of the literature will be sacrificed in favor of the adequate presentation of the results from the present work.

In Sect. 2, the PLP data and its processing which led to several detrended (unbiased) probability distributions of $1 \mathrm{~s}$ standard deviation of the measured irregularities in the ion density $\Delta N_{\mathrm{i}}$ will be described. Note that the parameter investigated by the present statistical studies is different from the ones analyzed by the references in the previous paragraphs. The reason for the present selection will also be provided in the next section. These probability distributions will be presented and described in Sect. 3 for several combinations of geophysical parameters (solar activity, altitude range, longitude sector, local time interval, season, and geomagnetic latitude interval). The same results will be discussed in Sect. 4, which will indicate that performing the statistical analysis with basis on $\Delta N_{\mathrm{i}}$ is relevant to scintillation-oriented work. Conclusions from the analysis will be listed in Sect. 5.

\section{Data description and processing}

PLP data are immediately available in individual daily files with $1 \mathrm{~Hz}$ resolution, but high-resolution data $(512 \mathrm{~Hz})$ are also available on request. Each $1 \mathrm{~s}$ record, corresponding to approximately $7.5 \mathrm{~km}$, associates the Universal Time (UT, s) to the corresponding average ion density $N_{\mathrm{i}}\left(\mathrm{cm}^{-3}\right)$, standard deviation of the ion density $\Delta N_{\mathrm{i}}\left(\mathrm{cm}^{-3}\right)$, ratio $\Delta N_{\mathrm{i}} / N_{\mathrm{i}}$, as well as satellite latitude (degrees), longitude (degrees), and altitude $(\mathrm{km})$. These records are obtained from the corresponding high-resolution data. Figure 1 displays an example of PLP data acquired during a single orbit (between consecutive crossings of the geographic equator) centered on local solar midnight. Its top panel shows the measured $1 \mathrm{~s}$ (green) and 1 min (black) average ion densities against the left vertical axis. This panel also shows the altitude of the satellite against the right axis (blue, with eclipse indicated by the heavily dotted line). The central panel shows spectrograms of the full $512 \mathrm{~Hz}$ data converted into units of distance with spectral power given in arbitrary units. The bottom panel represents the ground track of the satellite (red, heavily dotted during the eclipse) together with the magnetic equator (green).

The $1 \mathrm{~s}$ time series of the average ion density $N_{\mathrm{i}}$ allows different studies of irregularity structures with relatively large scale sizes. Indeed, considering the average C/NOFS satellite velocity and the well-known sampling theorem (Haykin, 1983), only structures with scale sizes greater than or equal to approximately $15 \mathrm{~km}$ are well characterized in the time series. On the other hand, each value of the standard deviation of the ion density $\Delta N_{\mathrm{i}}$ indicates the strength of the smallscale scintillation-causing irregularities in the corresponding $1 \mathrm{~s}$ interval, associated with the integrated power in the spectrograms of Fig. 1. According to Yeh and Liu (1982), under the conditions leading to a parabolic approximation to the scalar wave equation, one has

$$
\begin{aligned}
-2 i k \frac{\partial u}{\partial z}+\nabla_{\perp}^{2} u & =-k^{2}(\delta \varepsilon) u=4 \pi r_{\mathrm{e}}(\delta N) u \\
& =4 \pi r_{\mathrm{e}} \Delta N_{\mathrm{i}}(\delta \eta) u .
\end{aligned}
$$

In Eq. (1), $u$ is the complex amplitude of the transionospheric signal, $k$ is the free-space wavenumber, $z$ is aligned with the propagation direction, $\nabla_{\perp}^{2}$ is the transverse component of the Laplacian operator, $\delta \varepsilon$ and $\delta N$ are the zero-mean random permittivity and electron density fluctuations of the medium, and $r_{\mathrm{e}}=2.818 \times 10^{-15} \mathrm{~m}$ is the classical electron radius. Note that $\delta N$ has been recast in the form $\Delta N_{\mathrm{i}}(\delta \eta)$, where $\delta \eta$ is the normalized (zero-mean and unit-standard deviation) electron density fluctuations of the medium, to highlight the fundamental role of the absolute value of the standard deviation of the ion density $\Delta N_{\mathrm{i}}$ in the development of scintillation of transionospheric signals. Indeed, as stressed by Yeh and Liu (1982), the above is the basic equation upon which ionospheric scintillation theories are developed. 


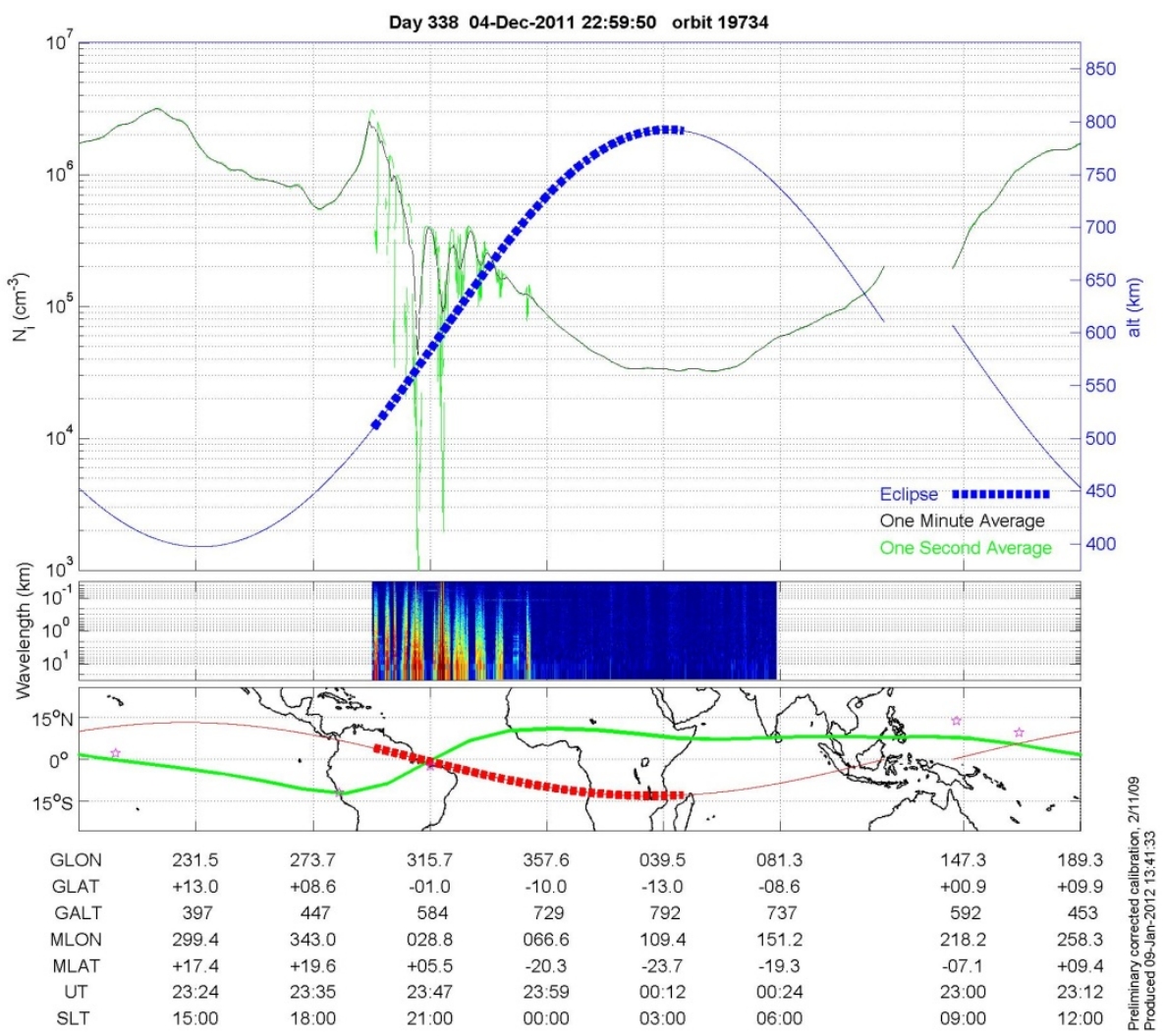

Figure 1. Orbit 19734, 4 December 2011. (top) One-minute average ionospheric density (black line); $1 \mathrm{~s}$ average density (green line); and satellite altitude (blue line). (middle) FFT from the $512 \mathrm{~Hz}$ PLP data. (bottom) Map with satellite trajectory (red) and magnetic equator (green). Thick dotted lines indicate when the C/NOFS satellite is in darkness.

The weak-scattering theory (Rufenach, 1975; Basu et al., 1976; Costa and Kelley, 1977; Rino, 1979) estimates the scintillation index $S_{4}$ of the signal received on the ground after propagating through an irregularity layer by the equation

$S_{4}=C\left(r_{\mathrm{e}} \lambda \sqrt{L L_{\mathrm{o}}}\right) \Delta N_{\mathrm{i}}\left(\sqrt{\pi} z_{\mathrm{f}} / L_{\mathrm{o}}\right)$

In the above equation, $S_{4}$ is the standard deviation of normalized received intensity $I /<I>$, where $I=|u|^{2}$ and $<$ $I>$ is the average value of $I, z_{\mathrm{f}}=(2 \lambda z)^{1 / 2}$ is the Fresnelscale size, $\lambda$ is the wavelength, $L$ and $z$ are the irregularity layer thickness and bottom height, respectively, and $L_{\mathrm{o}}$ is the outer-scale size of their power spectral density. Equation (2) assumes vertical propagation through the equatorial ionosphere and, due to slightly different power density spectral models assumed by the referenced authors, the constant $C$ varies from 0.6 to 2.0 . It should be stressed that the above result is only valid in the limits of the weak-scattering theory. The scintillation index $S_{4}$ asymptotically approaches unity in the strong-scattering regime (Yeh and Liu, 1982).

The right-hand sides of Eqs. (1) and (2) directly depend on $\Delta N_{\mathrm{i}}$, which explains the selection of this parameter for the present study. Note that most of the results from the references in Sect. 1 are based on a different parameter (the ratio $\left.\Delta N_{\mathrm{i}} / N_{\mathrm{i}}\right)$.

PLP data from 2 different years (1 October 200830 September 2009 and 1 January 2012-31 December 2012) were selected for analysis. The first data set corresponds to solar minimum conditions and the second one is as close to solar maximum conditions of solar cycle 24 as possible at the time of the analysis.

The results from the analysis show how the values of the standard deviation of the ion density $\Delta N_{\mathrm{i}}\left(\mathrm{cm}^{-3}\right)$ that exceed specified thresholds (to be defined in the next section) are statistically distributed, as functions of several combinations of the following parameters: (i) solar activity, (ii) altitude range, (iii) longitude sector, (iv) local time interval, (v) geomagnetic latitude interval, and (vi) season. The effects from the solar activity are represented by the differences between the results from the 2 selected years. Four localtime intervals ([18:00-21:00 LT), [21:00-24:00 LT), [00:0003:00 LT), [03:00-06:00 LT)) and four seasons (days 34-125 for the March equinox, 126-216 for the June solstice, 217307 for the September equinox and 308-33 for the December solstice) are considered.

To study the altitude distribution of the values of $\Delta N_{\mathrm{i}}$ that exceed the specified thresholds, four equal-size ranges are 
assumed: [400-500 km), [500-600 km), [600-700 km), and [700-800 km). Samples of $\Delta N_{\mathrm{i}}$ corresponding to satellite positions outside these intervals are discarded. This selection of altitude intervals, in addition to keeping uniformity, also takes into account the fact that, with age, the C/NOFS satellite no longer reaches the initial apogee $(850 \mathrm{~km})$, as observed in Fig. 1.

Nine uneven longitude sectors are selected: South American West $\left[270-300^{\circ}\right)$, South American East $\left[300-325^{\circ}\right)$, Atlantic $\left[325-343^{\circ}\right)$, African West $\left[343-030^{\circ}\right)$, African East $\left[030-060^{\circ}\right)$, Indian $\left[060-090^{\circ}\right)$, Asian $\left[090-130^{\circ}\right)$, Pacific West $\left[130-170^{\circ}\right)$, and Pacific East $\left[170-270^{\circ}\right)$. As indicated in the top panel of Fig. 2, the magnetic declination (red line and right vertical axis) remains relatively constant or displays a relatively constant longitudinal gradient in each of these sectors. Note that, with few exceptions (the Atlantic and the two Pacific sectors), the projection of the magnetic equator onto Earth (yellow line and left vertical axis) crosses land in most of the sectors. Thus, the above selection of longitude sectors is interesting, since topography has been listed (Friits and Alexander, 2003; Takahashi et al., 2009; Carrasco and Batista, 2012) as one of the possible drivers of gravity waves. These waves propagate vertically, reaching the bottom of the F layer to seed plasma instability processes that generate equatorial plasma bubbles and irregularities in the ionospheric density. The definition of the above longitude sectors may also permit the observation of seasonal and longitudinal effects on the occurrence of equatorial irregularities according to the alignment between the dusk terminator and the geomagnetic flux tubes (Abdu et al., 1981; Tsunoda, 1985). The bottom panel of Fig. 2 plots the strength of the magnetic field (yellow, left vertical axis) and indicates the 2 days of the year for approximate alignment between the magnetic field and the terminator line at the magnetic equator, for each longitude (red, right vertical axis). The meridional angle of the terminator was calculated in terms of the Julian day D by the approximate expression $\xi \approx 23.5^{\circ} \sin [\pi(D-81.0) / 182.5]$ (Burke et al., 2004a). The vertical red lines separate the adopted longitude sectors.

The magnetic latitude $\psi$ is approximately determined from the relationship $\tan \psi=(1 / 2) \tan I_{\mathrm{m}}$, where the magnetic inclination $I_{\mathrm{m}}$ is obtained from version 11 of the International Geomagnetic Reference Field model (IGRF-11) (International Association of Geomagnetism and Aeronomy and Working Group V-MOD, 2010) for the corresponding C/NOFS satellite position. To study the magnetic latitude distribution of the values of $\Delta N_{\mathrm{i}}$ that exceed the specified thresholds, three equal-size intervals are considered: $[-20$ to $\left.-10^{\circ}\right],\left[-5\right.$ to $\left.5^{\circ}\right]$, and $\left[+10\right.$ to $\left.+20^{\circ}\right]$. Irregularities outside these intervals are not considered by magnetic latitude studies. The second interval is centered at the magnetic equator and the other two should ordinarily include the peaks of the equatorial anomaly. For this study, the structure of the geomagnetic field (inferred from Figs. 1,2) and the orbital characteristics of the C/NOFS satellite (restricted to

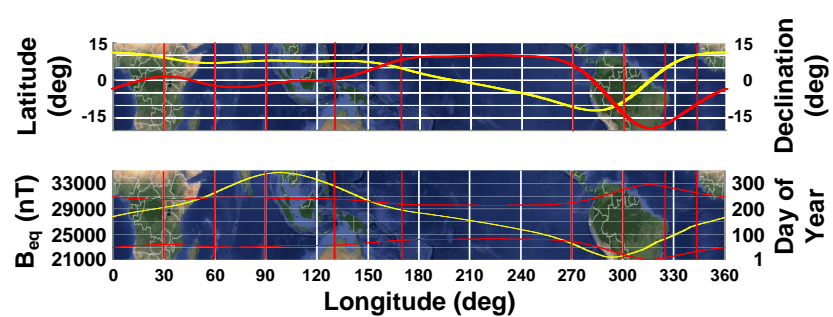

Figure 2. (top) Latitude of magnetic equator (yellow, left vertical axis) and corresponding declination (red, right vertical axis) for each longitude. (bottom) strength of the magnetic field (yellow, left vertical axis) and 2 days of the year for approximate alignment between the magnetic field and the terminator line at the magnetic equator for each longitude (red, right axis). The vertical red lines separate the adopted longitude sectors.

geographic latitudes less than approximately $13^{\circ}$ in absolute values) should be remembered. Based on the combination of the two features, it is easy to see that the C/NOFS satellite never transits through the first magnetic latitude interval $\left[-20\right.$ to $\left.-10^{\circ}\right]$ of the South American West longitude sector and the third magnetic latitude interval $\left[+10\right.$ to $\left.+20^{\circ}\right]$ from the Atlantic to the Pacific West longitude sectors.

In principle, the local time of the C/NOFS satellite is evenly distributed among the four selected intervals ([18:0021:00 LT), [21:00-24:00 LT), [00:00-03:00 LT), [03:0006:00 LT)), for evening hours. Uniformity is also applicable to yearly and seasonal distributions. In practice, missing samples and the restriction of data to the altitude interval [400$800 \mathrm{~km}$ ) distort uniformity somewhat.

In contrast with the uniformity characterized in the previous paragraph, it is evident that relative frequencies of observables in the longitude sectors are affected by their different widths.

Additionally, it should be observed that, also due to its orbital characteristics, the C/NOFS satellite is not neutral with respect to altitude and latitude measurements. Indeed, Fig. 3 shows the probability density functions (pdfs) of the C/NOFS satellite altitude and geographic latitude, resulting from the data files and predicted by a theoretical model (Fortes et al., 1999; ITU-R, 2001). Each of the curves in Fig. 3 is normalized in such a way that the pdf values at their break points add to one.

The top plot clearly shows the good agreement between the predicted and the initial altitude pdfs corresponding to the year 2008, as well as the progressive departure from them over the years. However, the good agreement between the predicted and the geographic latitude pdfs persisted throughout the years. It is also important to note that, as previously mentioned, the C/NOFS satellite transits through the selected altitude and geographic longitude intervals with different probabilities. For example, (1) the probability for the lower altitude interval $[400-500 \mathrm{~km}$ ) is greater than that for the one immediately above [500-600 km); and (2) the probability for 

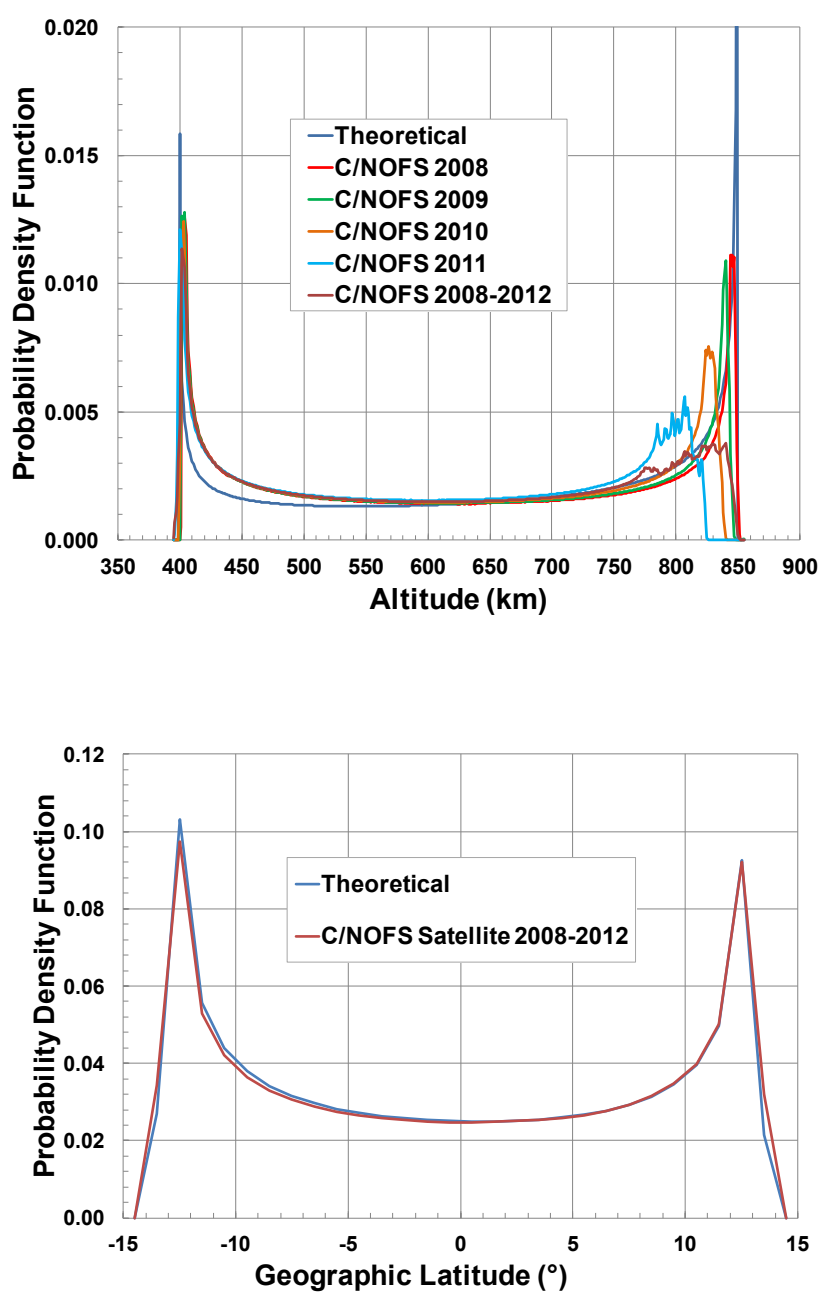

Figure 3. The pdfs of the C/NOFS satellite altitude (top) for different years and an extended period, as well as of the geographic latitude (bottom) for the same extended period, estimated from the data files. Also shown are the corresponding pdfs predicted by a theoretical model (Fortes et al., 1999; ITU-R, 2001) assuming nominal orbital parameters (inclination $13^{\circ}$, perigee and apogee approximately equal to 400 and $850 \mathrm{~km}$, respectively). Each of the above curves is normalized in such a way that the pdf values at their break points add to one.

the geographic latitude interval $\left[-13\right.$ to $\left.8^{\circ}\right)$ is greater than that for any other interval of the same length. From the lower panel of Fig. 1 and from Fig. 2, one can easily conclude that the absence of uniformity in the distribution of the geographic latitude of the C/NOFS satellite also assigns different probabilities to its presence in the selected magnetic latitude intervals.

Considering the need of taking the above observations into account, the detrended (unbiased) probability distribution of the values of $\Delta N_{\mathrm{i}}$ within each combination of parameters is obtained as follows. Initially, the probability of the occurrence of the C/NOFS satellite and the biased histogram of the values of $\Delta N_{\mathrm{i}}$ are separately determined for each member of a specified set of parameter classes. For example, the study of the probability distribution of the values of $\Delta N_{\mathrm{i}}$ with altitude ranges and longitude sectors (a total of $4 \times 9=36$ classes) generate 36 values of probability of the occurrence of the $\mathrm{C} / \mathrm{NOFS}$ satellite and the same number of biased histograms. Next, each value of a histogram is divided by the probability of the occurrence of the C/NOFS satellite in the corresponding class. Note that probabilities of certain occurrences of the C/NOFS satellite are equal to zero, as indicated by the last two sentences of the previous paragraph specifying magnetic latitude intervals. However, all the values of the corresponding histogram of $\Delta N_{\mathrm{i}}$ are also necessarily equal to zero and the above divisions are avoided in these cases. This is the only study where such a situation exists: only 20 out of 36 classes are available, due to the structure of the geomagnetic field and the orbital characteristics of the C/NOFS satellite. Finally, the set of resulting histograms are renormalized in such a way that all their values add to one. From the final set of histograms, the detrended (unbiased) probability of $\Delta N_{\mathrm{i}}$ exceeding each specified threshold is determined for each combination of parameters.

The above processing that leads to unbiased probabilities might be questionable if the probability of the presence of the C/NOFS satellite within one class were much smaller than that of another class. Therefore, particular care has been taken to confirm that, for each study, the set of probabilities of the presence of the C/NOFS satellite within each class did not excessively depart from uniformity. As an example of this preliminary analysis, one could think that the perigee of the C/NOFS satellite could show an extreme preference for a particular class of longitude sector and season of a single year. This situation could severely decrease the probability of the presence of the C/NOFS satellite within another class, making it very difficult to remove such a bias. Figure 4 confirms that the described situation did not occur. Its panels display the probability distribution functions of the presence of the C/NOFS satellite within each combination of longitude sector (on a per degree basis) and season of 2012 for the following altitude ranges: (a) [400-800 km), (b) [400$500 \mathrm{~km}$ ), and (c) [700-800 km). Thus, the results correspond to the full, lower and upper altitude ranges, respectively. This figure has been prepared with basis on the same $1 \mathrm{~s}$ files described in the first paragraph of the current section. Note that the variations of the probabilities in each of the three panels of Fig. 4, which also consider the eventual absence of data, are limited to only $18.37,16.04$, and $24.87 \%$, respectively. These results are consistent with the fact that the C/NOFS satellite's ascending node and perigee precess westward at the approximate rate of $24^{\circ}$ per orbit. Considering the number of 15 orbits per day, they visit all the longitude sectors every single day. In summary, after examining this and all the other situations of interest, no cases beyond the correction capability of the adopted procedures need to be reported. 

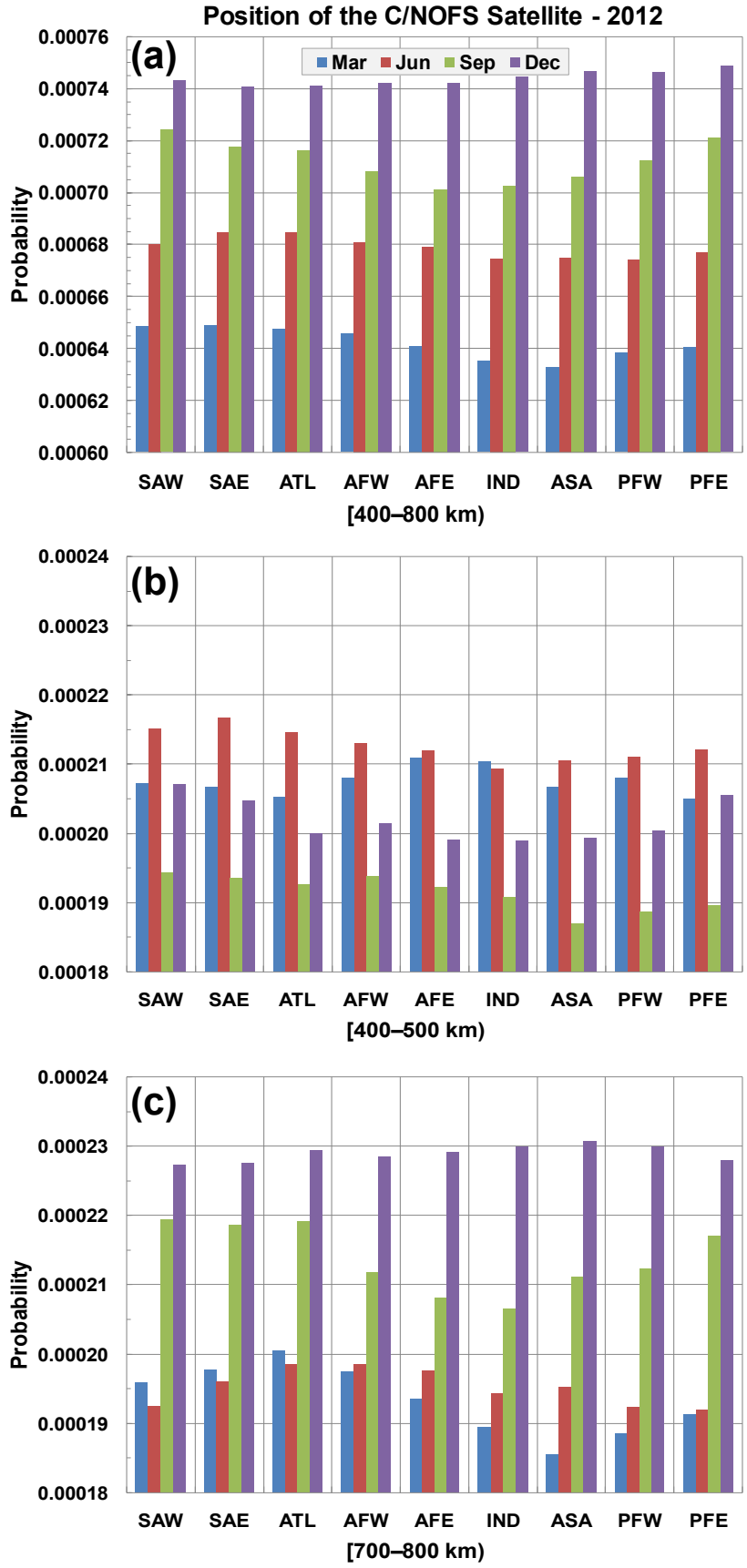

Figure 4. Probability distributions of the presence of the C/NOFS satellite within each combination of longitude sector (on a per degree basis) and season of 2012 for the altitude ranges: (a) [400$800 \mathrm{~km}),($ b) $[400-500 \mathrm{~km})$, and (c) [700-800 km). The maximum variations of the probabilities in the three panels are 18.37, 16.04, and $24.87 \%$, respectively.

\section{Results}

The totals of 11085676 and 12990898 samples of $\Delta N_{\mathrm{i}}$ resulted from the C/NOFS PLP measurements during the evening hours (between 18:00 and 06:00 LT) of the respective years 2008-2009 and 2012 in the altitude interval [400-800 km). The complementary cumulative distribution functions of $\log \left(\Delta N_{\mathrm{i}}\right)$ are plotted in Fig. 5 for these 2 years. The observed absolute maximum value of $\Delta N_{\mathrm{i}}$ corresponded to a negligibly low probability. For the current purposes, the maximum value of $\Delta N_{\mathrm{i}}$ exceeded with a significant probability was taken as $3.2 \times 10^{5} \mathrm{~cm}^{-3}\left[\log \left(\Delta N_{\mathrm{i}}\right)=5.5\right]$. Considering this reference as the maximum value, the threshold levels $\log \left(\Delta N_{\mathrm{i}}\right)>3.5$ and $\log \left(\Delta N_{\mathrm{i}}\right)>4.5$ were adopted. It should be stressed that, as indicated in Fig. 5, the global percentages of the above samples that exceeded the threshold $\log \left(\Delta N_{\mathrm{i}}\right)>3.5$ were approximately 0.72 and $11.40 \%$ for the years 2008-2009 and 2012, respectively, and that exceeding the threshold $\log \left(\Delta N_{\mathrm{i}}\right)>4.5$ was approximately $0.46 \%$ for the year 2012. There are 79816, 1480962 , and 59758 samples above the respective thresholds. The highest threshold level was exceeded during the solar-minimum year 2008 2009 with such a low probability that the corresponding distributions will not be shown.

For scintillation calculations, the estimation of $\Delta N_{\mathrm{i}}$ from in situ measurements is generally preceded by the subtraction of the least-square straight line from a space-limited batch of data. These processes act as a high-pass filter that limits the maximum scale size that is represented in the data batch, with a length defined with basis on two opposite constraints. It should be at least ten times longer than the Fresnel-scale size $z_{\mathrm{f}}=(2 \lambda z)^{1 / 2}$. However, it should be short enough to ensure that the irregularities in the record are homogeneous. It is well known that the power spectral density of equatorial ionospheric irregularities increases with the scale size (Yeh and Liu, 1982), as observed in the central panel of Fig. 1. Therefore, by increasing the length of the data batch from in situ measurements to correctly calculate the scintillation index $S_{4}$ as the operating frequency decreases, one allows larger and stronger structures to be considered in the estimation of $\Delta N_{\mathrm{i}}$. In an environment of homogeneous irregularities, the value of $\Delta N_{\mathrm{i}}$ would increase (Costa and Basu, 2002; C.-S. Huang, personal communication, December 2013). As an example, the estimation of $S_{4}$ values due to an irregularity layer with parameters $L=200 \mathrm{~km}, z=400 \mathrm{~km}$, and $L_{\mathrm{o}}=12.5 \mathrm{~km}$ at the nominal frequency $244 \mathrm{MHz}$ of SCINDA monitors (Groves et al., 1997) should be based on $10 \mathrm{~km}$ batches. The $\Delta N_{\mathrm{i}}$ samples available to the present study were obtained from $1 \mathrm{~s}$ or $7.5 \mathrm{~km}$ records. Ideally, they should have been characterized from one-third-longer records to estimate $S_{4}$ values at the SCINDA frequency. With the above caveats, Eq. (2) yields $S_{4} \approx 0.1$ and $S_{4} \approx 0.8$ for the assumed parameters of the irregularity layer and the thresholds $\log \left(\Delta N_{\mathrm{i}}\right)>3.5$ and $\log \left(\Delta N_{\mathrm{i}}\right)>4.5$, respectively.

According to Papoulis (1991), the 0.95 confidence interval for the estimation of the probability $p$ of an event by the relative frequency $x=k / n$ is approximately defined by 


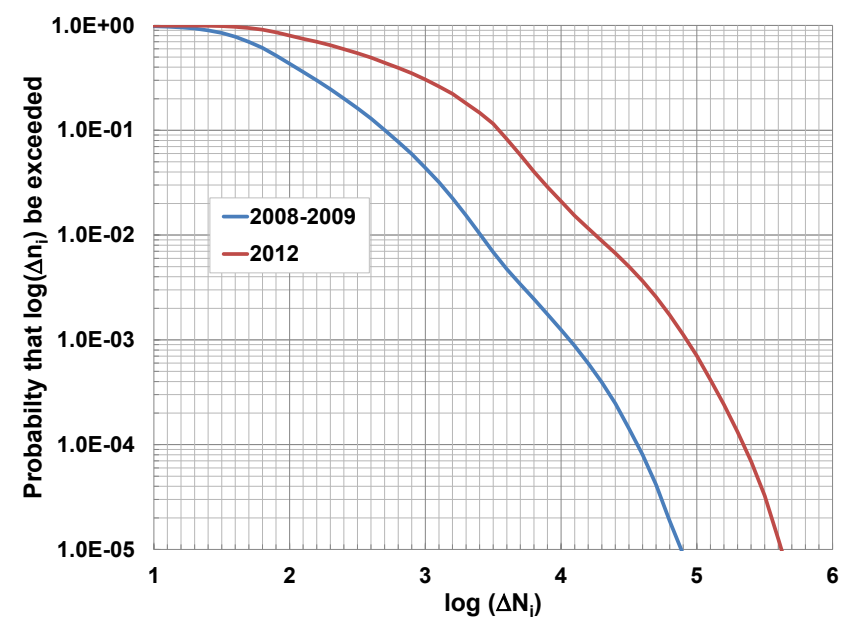

Figure 5. Complementary cumulative distribution functions of $\log \left(\Delta N_{\mathrm{i}}\right)$ in the altitude interval [400-800 km) for the years 20082009 (blue) and 2012 (red).

$$
\begin{aligned}
\frac{k}{n} & -\frac{1.967}{n} \sqrt{k}<p<\frac{k}{n}+\frac{1.967}{n} \sqrt{k} \\
& \rightarrow p \approx \frac{k}{n}\left(1 \pm \frac{1.967}{\sqrt{k}}\right)
\end{aligned}
$$

where $k$ is the number of occurrences of the event in a repeated trial, $n$ (greater than 100) is the total number of outcomes in the trial, and it is assumed that both $p$ and $x$ are much less than one. In other words, the relative 0.95 confidence interval for the estimation $p \approx k / n$ is approximately $\pm 196.7 / \sqrt{k} \%$. Kil and Heelis (1998) processed data from the AE-E mission recorded over a period of 3 years or more to ensure that $k$ was well above 100 in all the specified bins. Considering that data sets corresponding to 1 year have been processed separately to investigate solar activity effects, the threshold $k>100$ and the relative 0.95 confidence interval less than $\pm 20 \%$ will be accepted. All the probabilities associated with irregularities with $\log \left(\Delta N_{\mathrm{i}}\right)>3.5$ detected during 2012 meet these criteria of statistical significance. Cases of probabilities that do not meet the criteria will be identified as explained at the end of the next paragraph.

The next figures show detrended probability distributions of $\Delta N_{\mathrm{i}}$ in a common format for (a) 2008-2009 and $\log \left(\Delta N_{\mathrm{i}}\right)>3.5$, (b) 2012 and $\log \left(\Delta N_{\mathrm{i}}\right)>3.5$, and (c) 2012 and $\log \left(\Delta N_{\mathrm{i}}\right)>4.5$. For each figure, panels a and $\mathrm{c}$ share a common vertical full scale, which differs from that of panel $b$ by a factor equal to ten. Note that, to visually compare the probabilities in the three panels of each figure, the heights of the bars in panels a and c should also be divided by ten. Even acknowledging the large differences among the percentages in the previous paragraph and the vertical scales, the three sets of probability distributions will be presented and discussed. With the exception of Fig. 6, a common color code associated with the longitude sector is displayed inside panels a, being also applicable to the other panels. This color code should be read in sequence on line-by-line or top-tobottom bases. Each figure also has its own horizontal axis and title, displayed below panel $\mathrm{c}$ and shared with the other two panels. When applicable, each figure caption will indicate the smallest probability in panels a and $\mathrm{c}$ that meets the criteria established in the previous paragraph for statistical significance; smaller probabilities than these ones have been affected by undersampling and should be considered with care. It will be seen that these probabilities are always extremely small and irrelevant to the ensuing analyses. The information will not be provided when all probabilities in a panel meet the criteria.

Figure 6 displays the detrended probability distributions of $\Delta N_{\mathrm{i}}$ as functions of longitude sectors. That is, they approximately describe occurrences per degree in each of the longitude sectors. For the year 2008-2009, the South American East sector exhibits the largest probability of irregularities with $\log \left(\Delta N_{\mathrm{i}}\right)>3.5$, immediately followed by those of the South American West and Pacific East sectors. The sum of the three probabilities corresponds to approximately $56.30 \%$ of the total. The next set of intermediate probability values is formed by the African West, Atlantic and Pacific West sectors. The smallest probabilities are associated with the African East, Asian and Indian longitude sectors. Note that the ratio between the greatest and smallest probabilities is equal to 6.46 .

For the year 2012, the previous decreasing order of the probability of irregularities with $\log \left(\Delta N_{\mathrm{i}}\right)>3.5$ as a function of the longitude sector is essentially maintained, with some exceptions: the increases of the probabilities associated with the Atlantic and African West sector in relation to that of the Pacific East sector are observed. Figure $6 \mathrm{~b}$ also shows probabilities that are more evenly distributed among the longitude sectors. Indeed, the probabilities of the South American West, Atlantic, African West, and Pacific East are greater than $70 \%$ of the greatest one (associated with the South American East sector). The three smallest probabilities are related to the African East, Asian, and Indian sectors, but in the present case the ratio between the greatest and smallest probabilities is equal to only 1.95 .

For the same year and irregularities with $\log \left(\Delta N_{\mathrm{i}}\right)>4.5$, Fig. $6 \mathrm{c}$ confirms the greatest probability of the South American East sector by a large margin, immediately followed by that of the Atlantic, and next by those of the African West, South American West and Pacific East sectors. Only then one places the probability of the South American West sector. While the relative orders of the probabilities of the (i) South American West and South American East, (ii) African West and African East, and (iii) Pacific West and Pacific East sectors are maintained in the three plots; Fig. 6c shows an inversion in the order of the probabilities of the Asian and Indian sectors with respect to those of Fig. $6 a$ and b. For the 

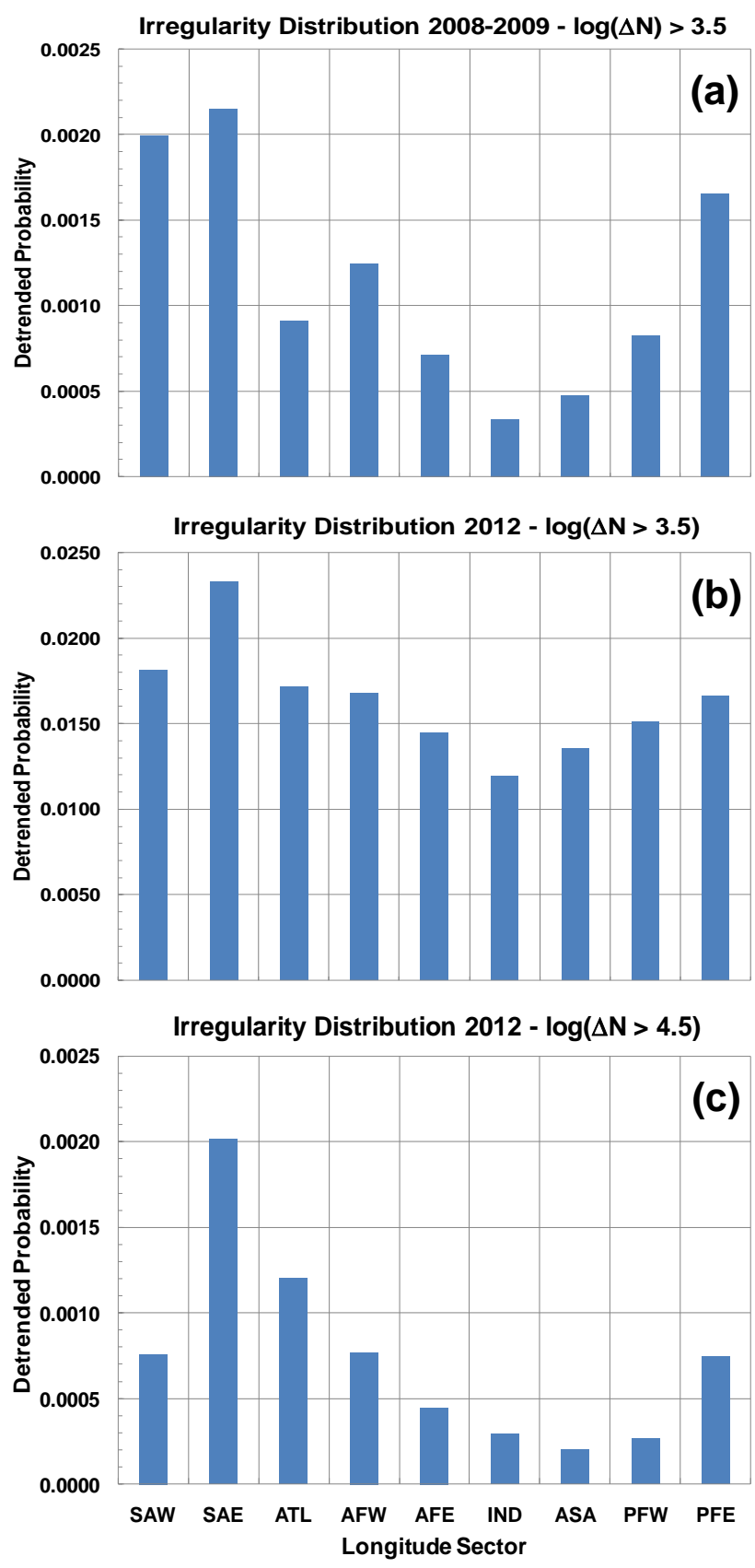

Figure 6. Detrended probability distributions of $\Delta N_{\mathrm{i}}$ as functions of longitude sectors for (a) 2008-2009 and $\log \left(\Delta N_{\mathrm{i}}\right)>3.5$, (b) 2012 and $\log \left(\Delta N_{\mathrm{i}}\right)>3.5$, (c) 2012 and $\log \left(\Delta N_{\mathrm{i}}\right)>4.5$.

strong irregularities with $\log \left(\Delta N_{\mathrm{i}}\right)>4.5$, the ratio between the greatest and smallest probabilities is equal to 9.96 .

Figure 7 exhibits the detrended probability distributions of $\Delta N_{\mathrm{i}}$ as functions of altitude ranges and longitude sectors. These distributions describe occurrences per degree in each of the longitude sectors and consider that the altitude of the C/NOFS satellite is not uniformly distributed among the four $100 \mathrm{~km}$ intervals. It is observed that the previous discussion related to Fig. 6a also applies to the lowest altitude range $[400-500 \mathrm{~km}$ ) of Fig. 7a. This panel additionally shows that the corresponding irregularities are rare above $500 \mathrm{~km}$, with the exception of those in the South American West and East sectors. Even for these two sectors, the probabilities decrease very fast (by factors approximately greater than five) between the two lowest altitude ranges.

For the year 2012, the irregularities with $\log \left(\Delta N_{\mathrm{i}}\right)>3.5$ reach the highest altitude range [700-800 km) with relatively small but non negligible probabilities for all the sectors. It is noted that the probabilities corresponding to the South American East sector remain the largest ones for all altitude ranges. The probability distribution for the lowest altitude range [ $400-500 \mathrm{~km}$ ) of Fig. $7 \mathrm{~b}$ is approximately the same as the one in Fig. 6b, except for the clear relative increases of the probabilities associated with the Pacific West and East sectors. It is interesting to note that, although the latter probability is greater than those of the South American West, Atlantic, and African West sectors for the lowest altitude range, this order is reversed for altitudes above $500 \mathrm{~km}$. Indeed, the probability associated with the Pacific East sector is the lowest of all sectors for the highest altitude range. Table 1 shows the results from a least-square fit of the exponential trend line $P r_{a}(h)=P r_{a} \exp (-a h)$ to the data from each longitudinal sector of Fig. $7 \mathrm{~b}$, where $h(\mathrm{~km})$ is the average height of each altitude range $(450,550,650$, and $750 \mathrm{~km}$, respectively). The first three columns of Table 1 show the longitudinal sectors and the associated values of the parameters $\operatorname{Pr}_{a}$ and $a$, respectively. The fourth column displays the values of the coefficient of determination $R^{2}$, which is a number from 0 to 1 that reveals how closely the values estimated by the trend line are to the measured data. A trend line is most reliable when its $R^{2}$ value is at or near 1 . The fifth column shows the probabilities $\operatorname{Pr}_{a}(450 \mathrm{~km})$ calculated by the trend line at $450 \mathrm{~km}$. The last column lists the values estimated from the data for the lowest altitude range $[400-500 \mathrm{~km}$ ), also displayed in the corresponding bin of Fig. 7b. The high $R^{2}$ values and a comparison between corresponding numbers in the last two columns of Table 1 indicate that, for each longitude sector, an exponential trend line provides a reasonable description of the decrease with height of the probability of irregularities with $\log \left(\Delta N_{\mathrm{i}}\right)>3.5$ observed during the year 2012.

Figure $7 \mathrm{c}$ shows that the irregularities with $\log \left(\Delta N_{\mathrm{i}}\right)>$ 4.5 detected during the year 2012 peak in the lowest altitude range $[400-500 \mathrm{~km}$ ) for all longitude sectors. Most are very rare above $600 \mathrm{~km}$. The same holds for the strong irregularities observed above $500 \mathrm{~km}$ from the African East to the Pacific West sectors. The exceptions are the ones associated with the South American East sector. Indeed, the probabilities related to this sector are clearly the greatest ones for each altitude range, up to $700 \mathrm{~km}$. The same order reversal with altitude among the probabilities associated with the Pacific East, South American West, and African West sectors also applies to the strong irregularities. Additionally, it should be 

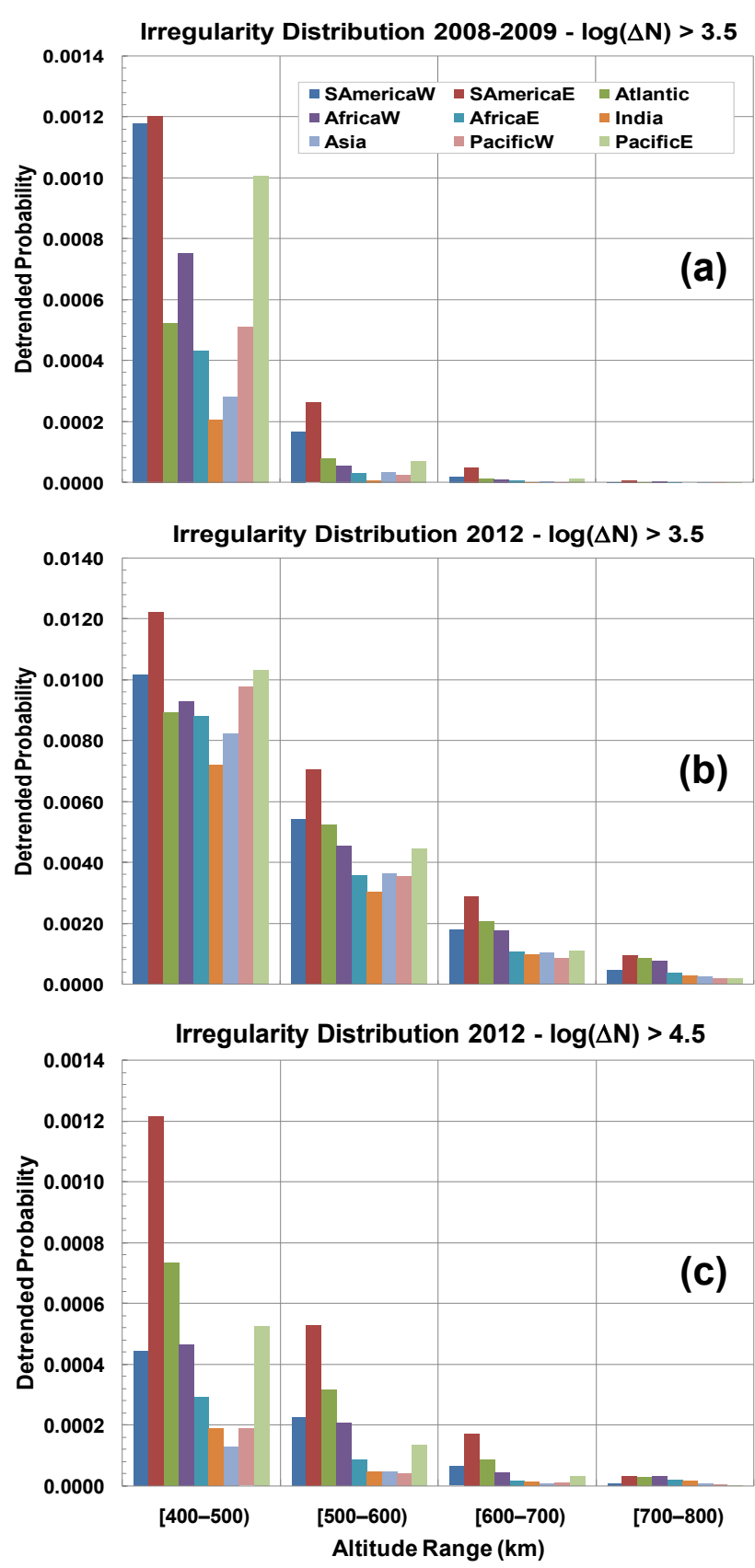

Figure 7. Detrended probability distributions of $\Delta N_{\mathrm{i}}$ as functions of altitude ranges and longitude sectors for (a) 2008-2009 and $\log \left(\Delta N_{\mathrm{i}}\right)>3.5$, (b) 2012 and $\log \left(\Delta N_{\mathrm{i}}\right)>3.5$, and (c) 2012 and $\log \left(\Delta N_{\mathrm{i}}\right)>4.5$. The smallest probabilities that meet the criteria for statistical significance are associated with the African East longitude sector and the altitude range $[600-700 \mathrm{~km}$ ) in (a) and the African East longitude sector and the altitude range [600-700 km) in (c), respectively.

noted that, differently from observations in the upper panels, the probability of strong irregularities in the Indian sector is greater than that in the Asian sector for the lowest altitude range.
Figure 8 shows the detrended probability distributions of $\Delta N_{\mathrm{i}}$ as functions of local time intervals and longitude sectors. These distributions describe occurrences per degree in each of the longitude sectors and consider that the position of the C/NOFS satellite is uniformly distributed among the four $3 \mathrm{~h}$ local time intervals. For the year 2008-2009, irregularities with $\log \left(\Delta N_{\mathrm{i}}\right)>3.5$ are extremely rare after 03:00 LT in all longitude sectors. It is interesting to observe that, from the South American East to the African East longitude sectors, the probabilities peak in the [21:00-24:00 LT) local time interval, being lower in the local time interval [18:0021:00 LT) by factors varying from 0.44 to 0.78 . For each longitude sector, the probability associated with the later local time interval [00:00-03:00 LT) is lower than (i) the corresponding one in the first local time interval, and (ii) its corresponding peak value, by a factor varying from 0.21 to 0.46 . Although the probability associated with the African West sector is greater than that of the Pacific sector for the [21:0024:00 LT) local time interval, inversions in this relationship are observed in the immediately earlier and later ones. A similar inversion is observed for the South American West and East sectors, considering the second and the first local time intervals.

As shown in Fig. 8b, the probabilities of irregularities with $\log \left(\Delta N_{\mathrm{i}}\right)>3.5$ for the year 2012 peak in the earliest local time interval [18:00-21:00 LT) and monotonically decrease with this parameter for all longitude sectors. Table 2 shows the result from a least-square fit of the exponential trend line $P r_{b}(t)=P r_{b} \exp (-b t)$ to the data from each longitudinal sector of Fig. 6b, where $t(\mathrm{~h})$ is the average value of each local time interval $(19.5,22.5,25.5$, and $28.5 \mathrm{~h}$, respectively). The first four columns of Table 2 show the longitudinal sectors and the associated values of the parameters $\operatorname{Pr}_{b}, b$, and $R^{2}$. The fifth column shows the probabilities $\operatorname{Pr}_{b}$ $(19.5 \mathrm{~h})$ calculated by the trend line at $19.5 \mathrm{~h}$. The last column lists the values estimated from the data for the lowest local time interval [18:00-21:00 LT), also displayed in the corresponding bin of Fig. $8 \mathrm{~b}$. The resulting $R^{2}$ values and a comparison between the corresponding numbers in the last two columns of Table 2 indicate that an exponential trend line provides an acceptable description of the decrease with local time of the probability of irregularities with $\log \left(\Delta N_{\mathrm{i}}\right)>3.5$ observed during the year 2012, particularly for the last four longitude sectors. The third and last columns of Table 2 indicate that (i) the greatest probability in the initial time interval, related to the South American East, decreases in time at a relative slow rate; and (ii) the smallest initial probabilities, related to the Indian and African East sectors, decrease in time at the second fastest and a moderate rate, respectively. The remaining sectors display initially intermediated probabilities. Two of these (for the Pacific West and East sectors) decrease in time at the slowest relative rates and another (for the South American West) at a moderate rate. The remaining three initial probabilities decrease in time at relatively fast 

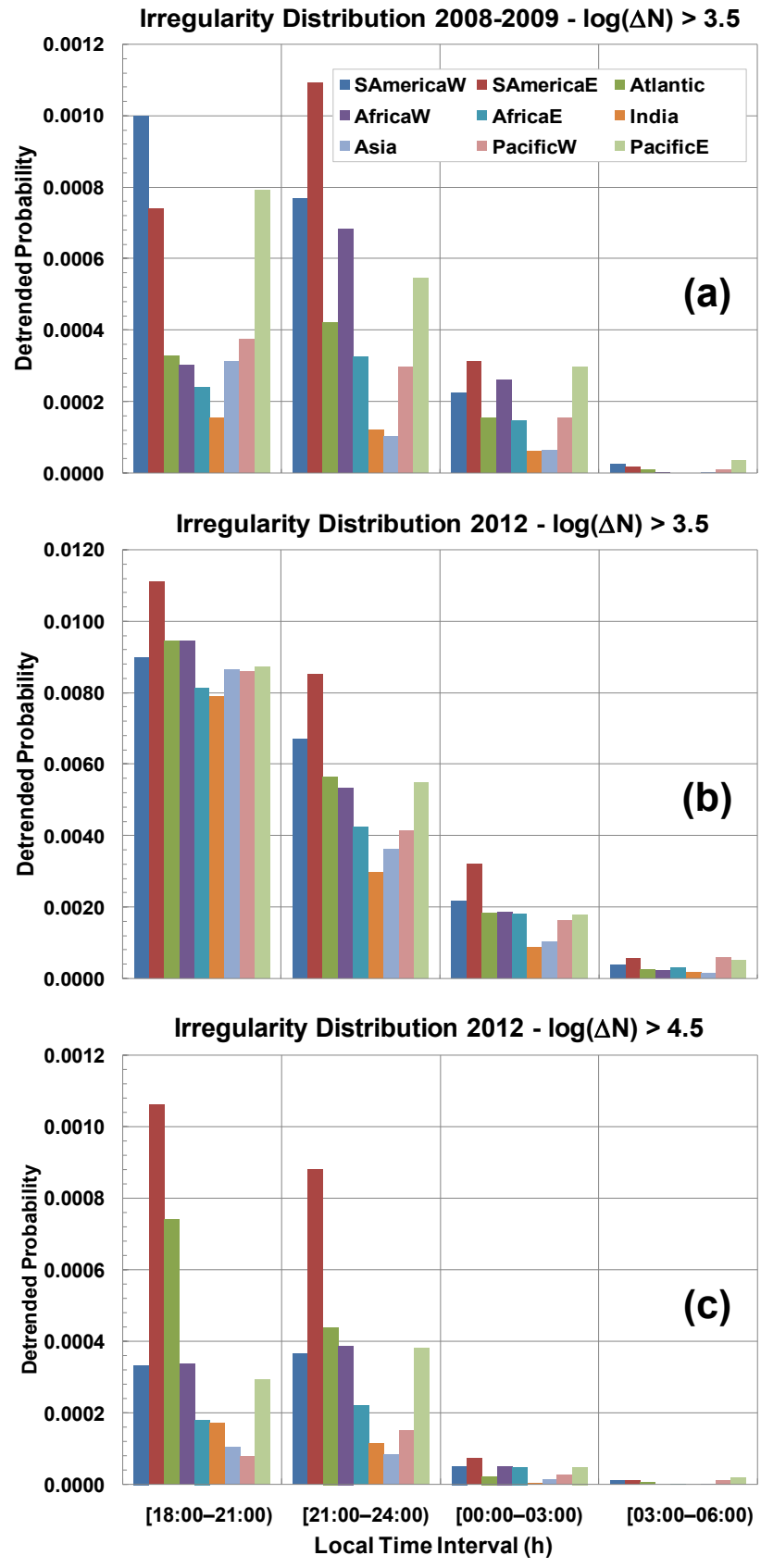

Figure 8. Detrended probability distributions of $\Delta N_{\mathrm{i}}$ as functions of local time intervals and longitude sectors for (a) 2008-2009 and $\log \left(\Delta N_{\mathrm{i}}\right)>3.5$, (b) 2012 and $\log \left(\Delta N_{\mathrm{i}}\right)>3.5$, and (c) 2012 and $\log \left(\Delta N_{\mathrm{i}}\right)>4.5$. The smallest probabilities that meet the criteria for statistical significance are associated with the Atlantic longitude sector and the local time interval [03:00-06:00 LT) in (a) and the Pacific West longitude sector and the local time interval [03:0006:00 LT) in (c), respectively.

rates (Atlantic and African West sectors) or the fastest rate (Asian sector).

Figure 8c shows that the irregularities with $\log \left(\Delta N_{\mathrm{i}}\right)>$ 4.5 detected during the year 2012 are very rare after 24:00 LT.
The probabilities corresponding to these strong irregularities in the South American East sector remain the largest ones for the two earliest local time intervals. Those corresponding to the South American East, Atlantic, Indian, and Asian sectors have their maximum values in the earliest and the other ones in the immediately following local time interval. However, some of the differences between corresponding probabilities in the two local time intervals are not substantial.

The detrended probability distributions of $\Delta N_{\mathrm{i}}$ as functions of magnetic latitude intervals and longitude sectors are displayed in Fig. 9. It should be remembered that, due to the geometry of the geomagnetic field and its orbital characteristics, the C/NOFS satellite never transits through the magnetic latitude interval $\left[-20\right.$ to $\left.-10^{\circ}\right]$ of the South American West longitude sector and the magnetic latitude interval $[+10$ to $+20^{\circ}$ ] from the Atlantic to the Pacific West longitude sectors. Figure 9a indicates that the probabilities of irregularities with $\log \left(\Delta N_{\mathrm{i}}\right)>3.5$ for the year 2008-2009 peak in the central $\left[-5\right.$ to $\left.+5^{\circ}\right]$ magnetic latitude interval for most longitude sectors, with the exception of that related to the Asian sector. Most of these peaks are prominent: the ratios between the probabilities of the central and lateral magnetic latitude intervals for the same longitude sector are generally greater than 2.40 and can reach 4.78 . The probabilities associated with the irregularities in the Pacific East sector display a roughly symmetrical distribution around the magnetic equator, in comparison to the clearly asymmetric distribution of those associated with the South American East sector.

Figure $9 \mathrm{~b}$ shows that the probabilities of irregularities with $\log \left(\Delta N_{\mathrm{i}}\right)>3.5$ for the year 2012 are more evenly distributed among the magnetic latitude intervals: the ratios between the probabilities of the central and lateral magnetic latitude intervals for the same longitude sector are always less 2.06. The probabilities of the irregularities in the South American East sector increase in the following order of magnetic latitude intervals: $\left[-10\right.$ to $\left.-20^{\circ}\right],\left[-5\right.$ to $\left.+5^{\circ}\right]$, and $\left[+10\right.$ to $\left.+20^{\circ}\right]$. For the Pacific East sector, this order is reversed: the probabilities associated with the first two intervals are equal to and greater than the one of the northern interval. Considering only the available data, the probabilities of these irregularities for the other longitude sectors peak in the $\left[-5\right.$ to $\left.+5^{\circ}\right]$ magnetic latitude interval.

Except for the difference between vertical scales, Fig. $9 \mathrm{~b}$ and $\mathrm{c}$ show that, for fixed longitude sectors, the respective distributions of probabilities display essentially similar dependences on the magnetic latitude interval. However, the same panels show different relative distributions of probabilities as functions of the longitude sector for each of the magnetic latitude intervals $\left[-10\right.$ to $\left.-20^{\circ}\right]$ and $\left[-5\right.$ to $\left.+5^{\circ}\right]$. For example, the greatest probabilities of irregularities with $\log \left(\Delta N_{\mathrm{i}}\right)>3.5$ detected in the magnetic latitude intervals $\left[-10\right.$ to $\left.-20^{\circ}\right]$ and $\left[-5\right.$ to $\left.+5^{\circ}\right]$ during the year 2012 are associated with the Pacific East and African West sectors, respectively. However, the probabilities of the irregularities 
Table 1. Results from the least square fit of the exponential trend line $P r_{a}(h)=P r_{a} \exp (-a h)$ to data of Fig. $7 \mathrm{~b}$. A trend line is most reliable when its $R^{2}$ value $\left(0 \leq R^{2} \leq 1\right)$ in the fourth column is at or near 1 . The column $P r_{\text {data }}$ lists the values of the bin [400-500 km) of Fig. $7 \mathrm{~b}$.

\begin{tabular}{lrrrrr}
\hline Longitude sector & $P r_{a}$ & $a$ & $R^{2}$ & $P r_{a}(450 \mathrm{~km})$ & $P r_{\text {data }}$ \\
\hline S. America West & 1.3358 & 0.0104 & 0.9755 & 0.0124 & 0.0102 \\
S. America East & 0.6566 & 0.0085 & 0.9798 & 0.0143 & 0.0122 \\
Atlantic & 0.3646 & 0.0080 & 0.9875 & 0.0100 & 0.0089 \\
Africa West & 0.4299 & 0.0084 & 0.9973 & 0.0098 & 0.0093 \\
Africa East & 1.2070 & 0.0108 & 0.9969 & 0.0094 & 0.0088 \\
India & 1.0674 & 0.0109 & 0.9934 & 0.0079 & 0.0072 \\
Asia & 2.1116 & 0.0119 & 0.9841 & 0.0100 & 0.0082 \\
Pacific West & 4.4543 & 0.0133 & 0.9921 & 0.0112 & 0.0098 \\
Pacific East & 5.6211 & 0.0135 & 0.9761 & 0.0129 & 0.0103 \\
\hline
\end{tabular}

Table 2. Results from the least square fit of the exponential trend line $P r_{b}(h)=P r_{b} \exp (-b t)$ to data of Fig. 8b. A trend line is most reliable when its $R^{2}$ value $\left(0 \leq R^{2} \leq 1\right)$ in the fourth column is at or near 1 . The column $P r_{\text {data }}$ lists the values of the bin [18:00-21:00 LT) of Fig. 8b.

\begin{tabular}{lrrrrr}
\hline Longitude Sector & $P r_{o}$ & $b$ & $R^{2}$ & $\operatorname{Pr}_{b}(19.5 \mathrm{~h})$ & $P r_{\text {data }}$ \\
\hline S. America West & 12.8513 & 0.3536 & 0.9157 & 0.0130 & 0.0090 \\
S. America East & 10.2346 & 0.3313 & 0.9008 & 0.0160 & 0.0111 \\
Atlantic & 29.5249 & 0.3950 & 0.9334 & 0.0133 & 0.0095 \\
Africa West & 38.0552 & 0.4075 & 0.9266 & 0.0135 & 0.0095 \\
Africa East & 10.6477 & 0.3555 & 0.9433 & 0.0104 & 0.0081 \\
India & 35.4666 & 0.4235 & 0.9875 & 0.0092 & 0.0079 \\
Asia & 82.1261 & 0.4558 & 0.9642 & 0.0113 & 0.0086 \\
Pacific West & 3.0212 & 0.2969 & 0.9954 & 0.0092 & 0.0086 \\
Pacific East & 5.4117 & 0.3186 & 0.9660 & 0.0108 & 0.0087 \\
\hline
\end{tabular}

with $\log \left(\Delta N_{\mathrm{i}}\right)>4.5$ in the South American East are the greatest in all three magnetic latitude intervals.

Figure 10 displays the distributions of the detrended probability of $\Delta N_{\mathrm{i}}$ as functions of seasons and longitude sectors. Based on the displayed results, Table 3 indicates, for each longitude sector and combination of year and threshold, the season that provides the minimum/maximum probability. All seasons that provide probabilities that are close (within 5\%) to the corresponding extreme value are listed between parentheses. For the cases with clearly distinct minimum and maximum probabilities, the second greatest probability is also indicated. It is observed that, for most longitude sectors, the probabilities reach their minimum values during the June solstice. In contrast with this general behavior, the probabilities of the irregularities in the Pacific West and East sectors, as well as those of the strong irregularities with $\log \left(\Delta N_{\mathrm{i}}\right)>4.5$ detected during 2012 in the African West, African East, and Asian sectors generally reach their relative minima during the December solstice. It is additionally observed that the relative minima of the first two sectors spread into the equinoxes. The isolated case of irregularities with $\log \left(\Delta N_{\mathrm{i}}\right)>3.5$ detected during 2012 in the South American West sector, which also reach their minimum probability during the December solstice, should be noted.
It is evident from Table 3 that, depending on the longitude sector and combination of year under study and threshold, the maximum probability is predominantly reached during the March equinox, followed by the December solstice. It is also noted in Fig. 8a that the irregularities with $\log \left(\Delta N_{\mathrm{i}}\right)>3.5$ detected in the South American East, Atlantic, and African East sectors are concentrated during the December solstice of the year 2008-2009, with $63.5,63.4$, and $50.0 \%$ of the total for each sector, respectively. The irregularities observed in the South American West, African West, Asian, Pacific West, and Pacific East sectors are less concentrated during the March equinox of the same year, with 41.5, 43.8, 41.3, 42.8 , and $48.2 \%$ of the total for each sector, respectively. The probabilities corresponding to the Indian sector are more evenly distributed among the seasons of the same year.

The last observation is also applicable to the probability distributions of the irregularities with $\log \left(\Delta N_{\mathrm{i}}\right)>3.5$ detected during 2012 in all the sectors, as indicated by Fig. 10b. Indeed, the maximum probability of these irregularities only reaches $33.5 \%$ of the corresponding total for each sector. Figure $10 \mathrm{~b}$ and the third column of Table 3 show that the probabilities of the irregularities observed in most longitude sectors reach their maximum values during the March equinox. With respect to other seasons, these maxima are not clearly dominant in the South Atlantic East, Atlantic, and 
Table 3. Seasonal (minimum/maximum/second greatest) detrended probability for each longitude sector from the data of Fig. 10 .

\begin{tabular}{llll}
\hline Longitude sector & 2008-2009; $\log \left(\Delta N_{\mathrm{i}}\right)>3.5$ & 2012; $\log \left(\Delta N_{\mathrm{i}}\right)>3.5$ & $2012 ; \log \left(\Delta N_{\mathrm{i}}\right)>4.5$ \\
\hline S. American West & $\mathrm{Jun} / \mathrm{Mar} / \mathrm{Sep}$ & Dec/Mar/Sep & $\mathrm{Jun} /(\mathrm{Mar}+\operatorname{Sep})$ \\
S. American East & Jun/Dec/Sep & Jun/Mar + Dec) & Jun/Dec/Mar \\
Atlantic & Jun/Dec/Mar & Jun/(Mar + Dec) & Jun/Dec/Mar + Sep) \\
African West & Jun/Mar/Sep & Jun/Sep/Mar & (Mar + Sep + Dec)/Jun \\
African East & Jun/Dec/Mar + Sep) & Jun/Mar/(Sep + Dec) & (Sep + Dec)/(Mar + Jun) \\
Indian & Jun/Dec/Mar + Sep) & Jun/Mar/(Sep + Dec) & Jun/Mar/Sep \\
Asian & Jun/Mar/Sep & Jun/Mar/Sep & Dec/Mar/Sep \\
Pacific West & Dec/Mar/Sep & Jun/Mar/Sep & Dec/Jun/Mar \\
Pacific East & Dec/Mar/Sep & Dec/(Mar +Sep) & Dec/Sep/Mar \\
\hline
\end{tabular}

Pacific East sectors. It is also observed that the probability related to the African West sector has its maximum value during the September equinox.

As indicated by Fig. 10c and the last column of Table 3, the probabilities of the strong irregularities with $\log \left(\Delta N_{\mathrm{i}}\right)>4.5$ detected during the year 2012 in the South American West, Indian, and Asian sectors reach their maximum values in the March equinox. The maximum probability of the first of these sectors is essentially equal to that of the September equinox, with their combination adding to $77.6 \%$ of the corresponding total. The maxima of the other two sectors are concentrated in the initially quoted season, amounting to 46.3 and $57.5 \%$ of their totals, respectively. Differently from this behavior, the probabilities for the South American East and Atlantic sectors reach their maxima during the December solstice, while the one for the Pacific sector maximizes during the September equinox of the same year. These isolated maxima correspond to $41.7,41.7$, and $43.9 \%$ of the totals for the sectors, respectively. Finally, the probabilities of the strong irregularities in the African West, African East, and Pacific West sectors reach their maximum values in the June solstice. The probabilities in the first of these sectors are almost uniformly distributed among the four seasons. The maximum probability of the second sector is extremely close to that of the March equinox and their sum is equal to $60.8 \%$ of the corresponding total. In contrast, the isolated maximum of the third sector represents $38.9 \%$ of the respective total.

\section{Discussion}

It is well accepted that large-scale equatorial ionospheric irregularities are created by the action of the generalized Rayleigh-Taylor (R-T) instability that could be initiated under favorable conditions at the bottom of the $\mathrm{F}$ region shortly after sunset. Initially, seeds from different origins linearly grow with the rate (Burke et al., 2004a, and references therein)

$\gamma_{\mathrm{R}-\mathrm{T}}=\frac{\Sigma_{\mathrm{F}}^{\mathrm{P}}}{\Sigma_{\mathrm{F}}^{\mathrm{P}}+\Sigma_{\mathrm{E}}^{\mathrm{P}}}\left(V_{\perp}+U_{\perp}^{\mathrm{P}}+\frac{g}{v_{\text {in }}}\right) \frac{1}{L_{n}}-R$
In the above equation, $\Sigma_{\mathrm{F}}^{\mathrm{P}}$ and $\Sigma_{\mathrm{E}}^{\mathrm{P}}$ are the contributions of the $\mathrm{F}$ and $\mathrm{E}$ regions to the Pedersen conductivity integrated along the flux tube of magnetic field lines with apex at the bottom of the $\mathrm{F}$ region. The following variables should be calculated at the apex of the flux tube (at the magnetic equator): $V_{\perp}$ is the vertical component of the drift $\boldsymbol{E} \times \boldsymbol{B}_{\text {eq }} / B_{\text {eq }}^{2}$, which is perpendicular to the magnetic field at the magnetic equator; $B_{\text {eq }}$ is the strength of the magnetic field; and $g$ is the acceleration due to gravity. Additionally, $U_{\perp}^{\mathrm{P}}$ is the vertically downward component of neutral wind velocity perpendicular to the magnetic field, weighted by $\left(\Sigma_{\mathrm{F}}^{\mathrm{P}}+\Sigma_{\mathrm{E}}^{\mathrm{P}}\right) ; \nu_{\text {in }}$ is the flux-tube integrated $\mathrm{F}$ region ion-neutral collision frequency, weighted by the number density; $L_{n}$ is the scale length of the vertical gradient of the flux-tube integrated plasma density in the $\mathrm{F}$ region; and $R$ is the recombination rate integrated along the flux tube. Eastward electric field components yield positive $V_{\perp}$ values, raising the $\mathrm{F}$ layer and consequently decreasing $v_{\text {in }}$. In this case, the first and third terms between parentheses in Eq. (4) enhance the growth rate. The opposite occurs for westward components of the electric field. Since $V_{\perp}$ is inversely proportional to $B_{\text {eq }}$, Eq. (4) indicates that these effects on the growth rate should be anticorrelated with the longitudinal dependence of the field strength of the magnetic field observed in the lower panel of Fig. 2, for a constant value of the eastward electric field (Huang et al., 2001; Burke et al., 2004a, b). Equation (4) also indicates that the growth rate increases when $\Sigma_{\mathrm{E}}^{\mathrm{P}}$ decreases. This process is more effective when sunset is simultaneous at the conjugate E layers of the flux tube (Abdu et al., 1981; Tsunoda, 1985). In other words, this situation occurs when the dusk terminator and the magnetic flux tubes are aligned, at 2 different days in the year for each longitude, as shown in the lower panel of Fig. 2. During the nonlinear evolution of the R-T instability, depleted regions of plasma (equatorial plasma bubbles, EPBs) rise to the topside ionosphere and generally drift eastward, while a hierarchy of secondary instabilities cascade energy into irregularities with decreasing-scale sizes, until being quenched.

After this brief outline of a basic theory that explains the generation and evolution of irregularities in the equatorial ionospheric $\mathrm{F}$ region, the results described in Sect. 3 will 


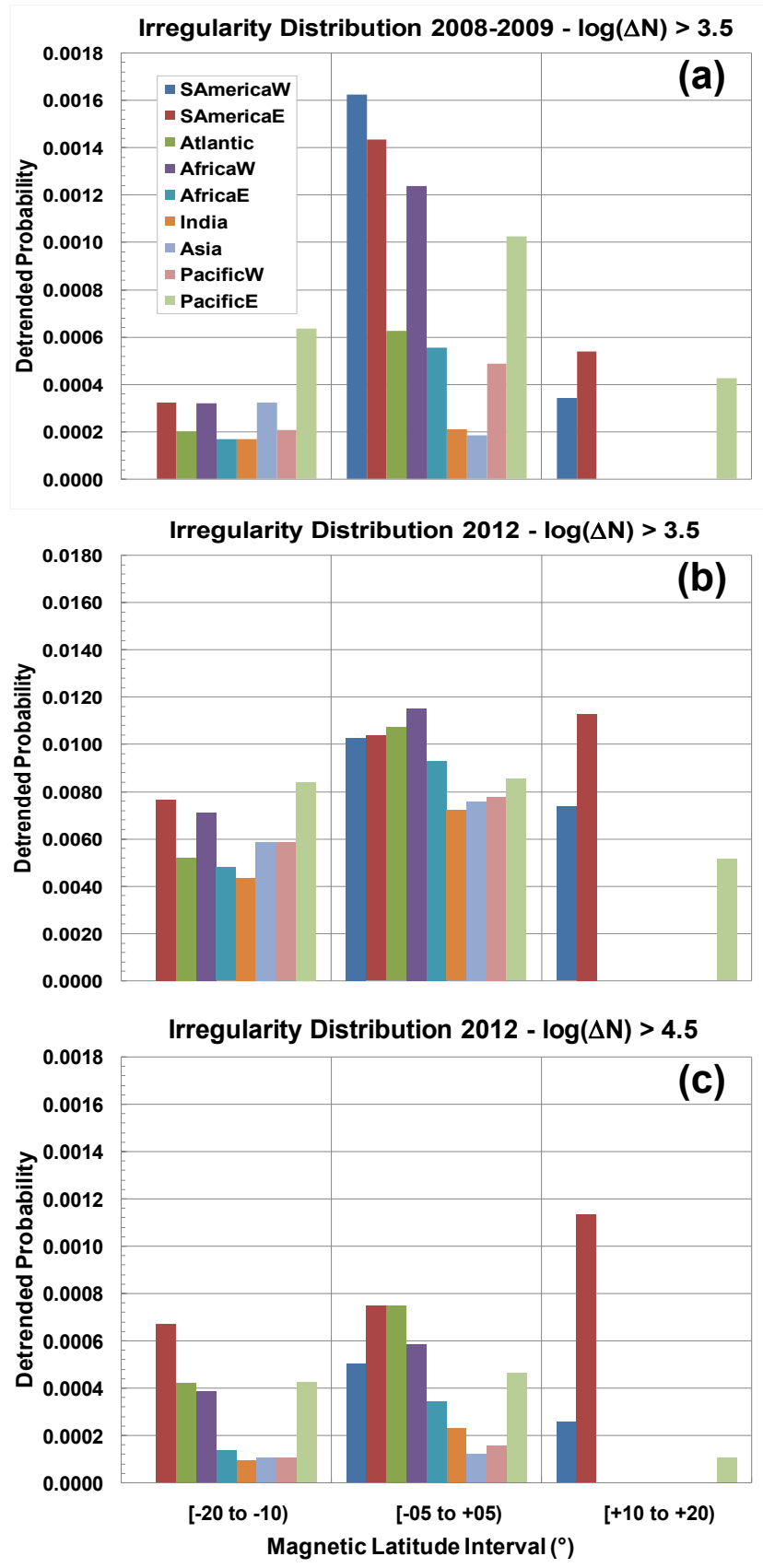

Figure 9. Detrended probability distributions of $\Delta N_{\mathrm{i}}$ as functions of magnetic latitude intervals and longitude sectors for (a) 20082009 and $\log \left(\Delta N_{\mathrm{i}}\right)>3.5$, (b) 2012 and $\log \left(\Delta N_{\mathrm{i}}\right)>3.5$, and (c) 2012 and $\log \left(\Delta N_{\mathrm{i}}\right)>4.5$. Note that the C/NOFS satellite, due to its orbital characteristics and the geometry of the geomagnetic field, never transits through the magnetic latitude interval $\left[-20\right.$ to $\left.-10^{\circ}\right]$ of the South American West longitude sector and the magnetic latitude interval $\left[10\right.$ to $20^{\circ}$ ] from the Atlantic to the Pacific West longitude sectors.

be discussed and related to some of those from the previous satellite programs, where applicable. Before doing so, it should be remarked that simple substitutions of observables
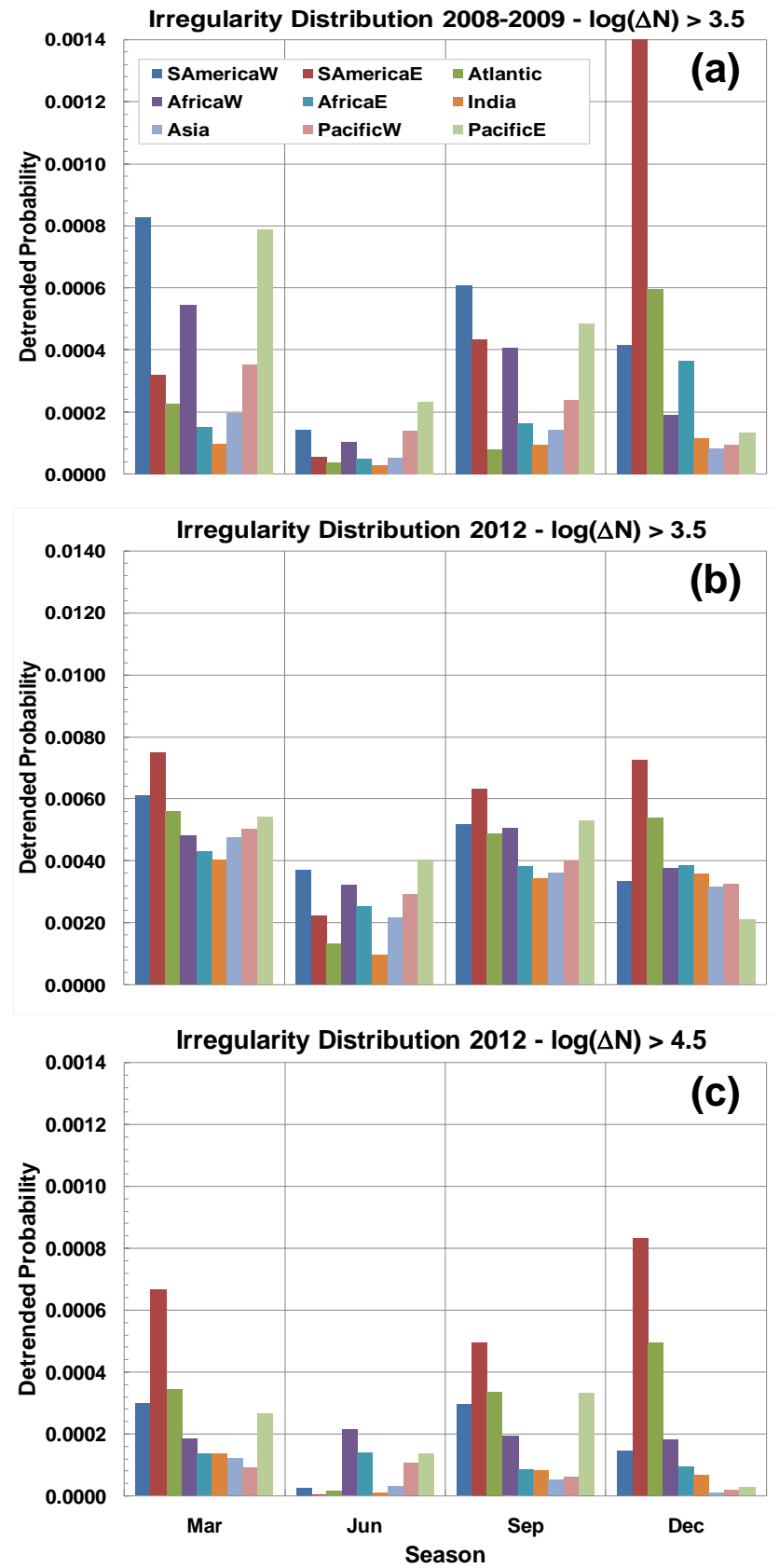

Figure 10. Detrended probability distributions of $\Delta N_{\mathrm{i}}$ as functions of seasons and longitude sectors for (a) 2008-2009 and $\log \left(\Delta N_{\mathrm{i}}\right)>$ 3.5, (b) 2012 and $\log \left(\Delta N_{\mathrm{i}}\right)>3.5$, and (c) 2012 and $\log \left(\Delta N_{\mathrm{i}}\right)>$ 4.5. The smallest probability that meets the criteria for statistical significance is associated with the Asian longitude sector and the December solstice in (c).

and thresholds allowed the same processing tool to provide the results in the previous and present sections.

Figure 11a displays the detrended probability distribution of $\Delta N_{\mathrm{i}} / N_{\mathrm{i}}>1 \%$ recorded during 2012 as a function of longitude sectors. In the other panels, the distributions of $\Delta N_{\mathrm{i}} / N_{\mathrm{i}}>1 \%$ are shown as functions of longitude sectors 
and local time intervals (panel b), magnetic latitude intervals (panel c), and seasons (panel d). These results and threshold are based on exactly or approximately the same parameter adopted by Kil and Heelis (1998) or Su et al. (2006), respectively. It has been remarked in Sect. 1 that Huang et al. (2002) and Burke et al. (2004a, b) based their analyses on a different parameter. In principle, differences between corresponding results could be due to the respective measurement periods and the associated solar activities, orbital characteristics of the satellites, instrumentation, processing techniques developed by different authors, etc. The scale-size range observed by the present study intersects with that by Kil and Heelis (1998) but it is disjoint with the ones sampled by $\mathrm{Su}$ et al. (2006), Huang et al. (2002), and Burke et al. (2004a, b).

It is observed that, as functions of longitude, the probability distribution in Fig. 11a and $B_{\text {eq }}$ in the lower panel of Fig. 2 are roughly anticorrelated. However, the minimum of $B_{\text {eq }}$ occurs at $280^{\circ}$, while the maximum detrended probability of $\Delta N_{\mathrm{i}} / N_{\mathrm{i}}>1 \%$ is associated with the South American East longitude sector $\left[300-325^{\circ}\right)$. The ratios between the extreme values of probabilities in Fig. 11a and field strengths in the lower panel of Fig. 2 are different: $0.0221 / 0.0077=2.87$ and $34691 / 21467=1.62$, respectively.

Even though a long list of sources of potential differences between the results in Fig. 11b-d and the corresponding ones from the previous satellite programs has been presented, the agreements between them are actually good. Indeed, Fig. 4 of the paper by Kil and Heelis (1998) shows that the occurrence probability of $\Delta N_{\mathrm{i}} / N_{\mathrm{i}}>1 \%$ above $350 \mathrm{~km}$ increased rapidly after sunset, reaching a flat maximum between 20:00 and 23:00 LT. It then decreases linearly with local time to essentially zero by sunrise (08:00 LT). These observations are consistent with the ones derived from Fig. 10 of the paper by $\mathrm{Su}$ et al. (2006) for $\mathrm{Kp}<3$ and from Fig. 11b. In particular, it is noted that the three plots indicate appreciable occurrence probabilities of $\Delta N_{\mathrm{i}} / N_{\mathrm{i}}>1 \%$ in the local time interval [03:00-06:00 LT).

Figure 10 of the paper by Kil and Heelis (1998) shows the occurrence probabilities of $\Delta N_{\mathrm{i}} / N_{\mathrm{i}}>1 \%$ as functions of magnetic latitude, for irregularities detected above $350 \mathrm{~km}$ during each season. They are strongly peaked near the dip equator and decrease rapidly with magnetic latitude. Most of the values are negligible for latitudes greater than $20^{\circ}$. All distributions show asymmetries about the equator that depend on season. They rise more steeply for magnetic latitudes on the summer side and less steeply at the conjugate location, with peaks displaced to the summer side. Figure 9 of the paper by Burke et al. (2004b) shows occurrence rates of EPBs in March and April of the years 2000 and 2002 as functions of the magnetic latitude, using ROCSAT- 1 data. The plots associated with the 2 years display Gaussian-like distributions centered at the magnetic equator. The bell-shaped observations are confirmed in Fig. 5 of the paper by Su et al. (2006), based on ROCSAT-1 data from the years 1999 to 2004. These authors observed slight asymmetries in the distributions around the magnetic equator for the two solstice seasons in contrast with more symmetrical ones for the two equinox seasons. The summer hemisphere exhibits a slightly higher occurrence rate than the winter one. Even so, any asymmetries in the distributions reported by Burke et al. (2004b) and Su et al. (2006) are clearly less pronounced than the ones displayed by Kil and Heelis (1998). All the detrended probabilities distributions in Fig. 11c are consistent with the ones resulting from ROCSAT-1 data. Indeed, for each longitude sector, the biggest ones are associated with the central magnetic latitude interval $\left[-5\right.$ to $\left.+5^{\circ}\right)$. The only two distributions that could be fully characterized using C/NOFS PLP data, associated with the South American East and Pacific East longitude sectors, display discrete asymmetries around the magnetic equator.

The previous authors used different horizontal (longitudinal) axes in their plots of the longitude dependence of the occurrence rates of $\Delta N_{\mathrm{i}} / N_{\mathrm{i}}$ or EPBs for each season. Figure 11 of the paper by Kil and Heelis (1998) and Figs. 2 and 1 of the papers by Burke et al. (2004a, b), respectively, adopted a longitudinal axis which varied from 0 to $360^{\circ}$, while the one used in Fig. 5 of the paper by Huang et al. (2001), restricted to the December solstice, and Fig. 4 of the paper by $\mathrm{Su}$ et al. (2006) began and ended at $90^{\circ}$. That in Fig. 11d begins and ends at $270^{\circ}$. Fortunately, the application used to prepare Fig. 11d allows the horizontal axis to be circularly shifted, facilitating the comparison of the present with the previous results. Taking into account that the longitude sectors of Fig. 11d are relatively wide and uneven, while the resolution used in all the previous reports were fine and uniform, it can be said that the agreement is good, particularly during the solstices. However, the longitudinal resolution of Fig. 11d does not allow observing the narrow secondary peak centered at $70^{\circ}$ in longitude observed by Huang et al. (2001) and Su et al. (2006) during the December solstice. This peak was not observed by the other authors either.

It is important to add that, with few exceptions, the longitudinal sector distributions in all the classes of Fig. 11b-d resemble the one in Fig. 11a. Thus, they are roughly anticorrelated with the longitudinal dependence of $B_{\text {eq }}$ in the lower panel of Fig. 2, as proposed by Huang et al. (2001). Additionally, Fig. 11d shows that the probabilities associated with the South American East, South American West and Atlantic longitude sectors, which cover the interval $\left[270-343^{\circ}\right.$ ), maximizes during the December solstice, in good agreement with the model proposed by Tsunoda (1985). Indeed, the angles between the dusk terminator and the magnetic flux tubes are small during this season, as shown in the lower panel of Fig. 2. However, it should be remembered that relatively wide longitude sectors and seasons have been used by the present work. The theories proposed by Tsunoda (1985) and Huang et al. (2001) were submitted to closer scrutiny by Burke et al. (2004a) by classifying DMSP data into a finer 1 -month $\times 15^{\circ}$ longitude grid. They concluded that, out of (24 longitudes $\times 2$ days/longitude) 48 days, 18 and 44 of the 


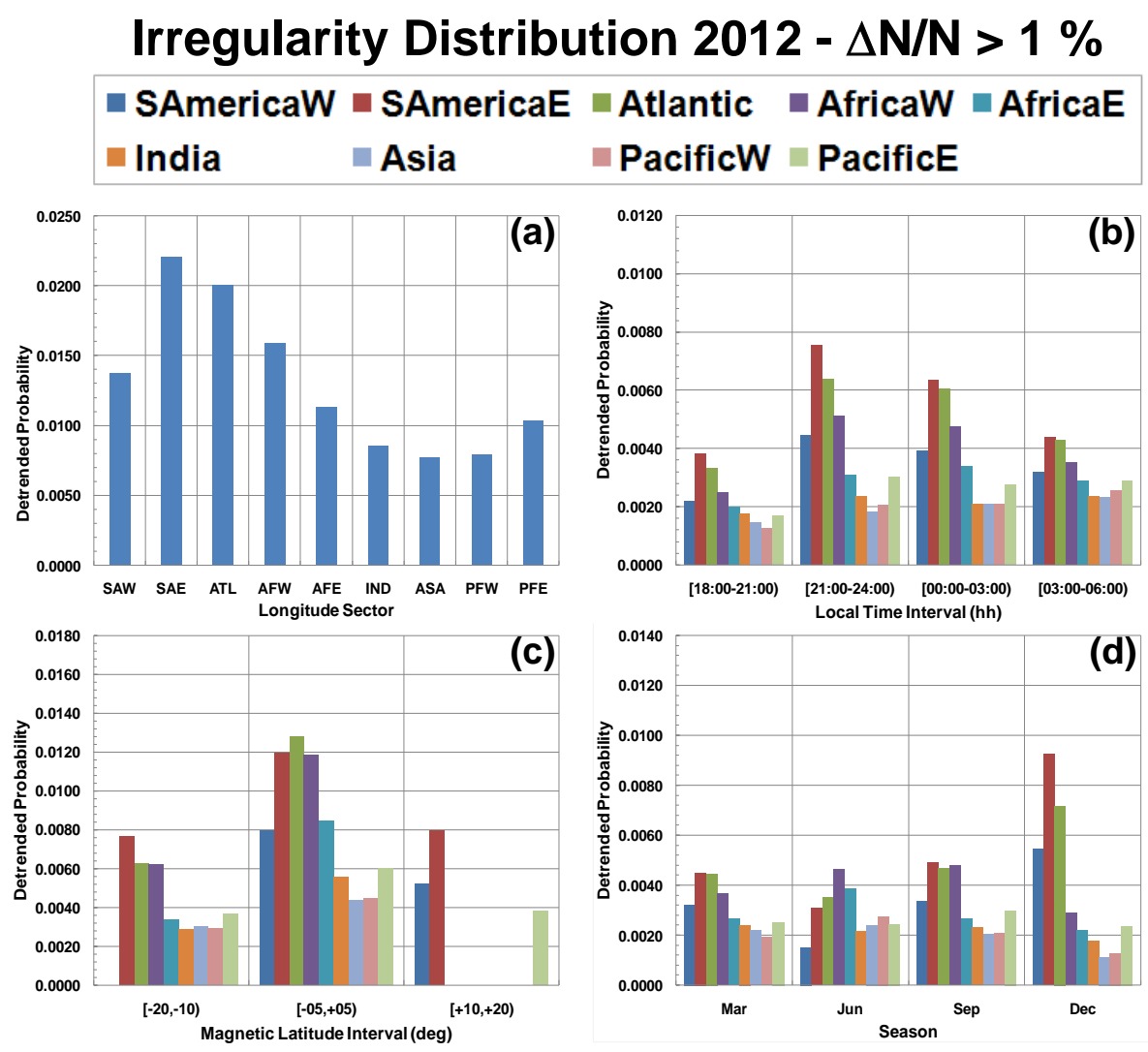

Figure 11. Detrended probability distributions of $\Delta N_{\mathrm{i}} / N_{\mathrm{i}}>1 \%$ recorded during 2012 as functions of (a) longitude sectors, (b) local time intervals, (c) magnetic latitude intervals, and (d) seasons. The vertical axes coincide with those of the central panels of Figs. 6, 8, 9, and 10, respectively.

maxima in the observed EPB occurrence rate were found within 2 weeks and 1 month of the days predicted by the Tsunoda model (1985), respectively. They also observed the general trend proposed by Huang et al. (2001), but that the extremes of the EPB rates were displaced in longitude in relation to those of $B_{\text {eq }}$. Furthermore, they noted an asymmetric pattern in the region of weak magnetic fields, with greater EPB rates associated with positive longitudinal gradients of $B_{\text {eq. }}$.

In summary, the above discussion has established that the results in Fig. 11 are in good agreement with the corresponding ones from the previous satellite programs.

If the denominator in the left-hand side of $\Delta N_{\mathrm{i}} / N_{\mathrm{i}}>$ $1 \%$ were the ambient ion density, one could be tempted to assume that $\Delta N_{\mathrm{i}}>N_{\mathrm{i}} / 100$ and use a "typical" value for $N_{\mathrm{i}}$ to estimate a corresponding threshold for $\Delta N_{\mathrm{i}}$. For example, a $400 \mathrm{~km}$ circular-orbiting satellite would measure $N_{\mathrm{i}} \approx 10^{6} \mathrm{~cm}^{-3}$, during solar maximum conditions, yielding $\Delta N_{\mathrm{i}}>10^{4} \mathrm{~cm}^{-3}$ or $\log \left(\Delta N_{\mathrm{i}}\right)>4.0$. In this case, the results from Fig. 11 and the previous satellite programs could be reinterpreted in terms of the distributions of $\Delta N_{\mathrm{i}}$, decreasing the importance of the results from the previous section. However, the above conversion would be a rough one, for ignoring space and time variations of the ambient ion density along circular orbits. Indeed, the ambient value of $N_{\mathrm{i}}$ changes across the equatorial anomaly region, from early to late evening hours, etc. As illustrated by the $1 \mathrm{~min}$ average ion density curve in the upper panel of Fig. 1 between 23:41 and 23:59 UT, the conversion would be even less straightforward in the case of the C/NOFS satellite, due to the large excursion of the ambient plasma density within the covered altitude range. Moreover, $N_{\mathrm{i}}$ is not the ambient ion density in any of the discussed results from the AE-E, ROCSAT-1 and C/NOFS programs - it is actually the average ion density over a generally short data batch. Their lengths, quoted in Sect. 1 for the previous satellite programs and in Sect. 2 for the C/NOFS data, vary between 75 and $7.5 \mathrm{~km}$. Several of these batches can be situated in the interior of a single equatorial plasma bubble, where $N_{\mathrm{i}}$ may experience extreme changes. The same upper panel of Fig. 1 shows that the $1 \mathrm{~min}$ average ion density decreased by approximately one order of magnitude from the edges to the bottom of the $900 \mathrm{~km}$ plasma bubble that ends at 23:47 UT, while the satellite altitude only changed by $30 \mathrm{~km}$.

Therefore, the dependence of the detrended probability distributions of $\Delta N_{\mathrm{i}}$ on geophysical parameters, which are 
directly relevant to scintillation-oriented studies, cannot be accurately inferred from those of $\Delta N_{\mathrm{i}} / N_{\mathrm{i}}$. In combination with the arguments in the preceding paragraph, this single example clearly establishes the differences between the probabilities obtained from the analyses of the two parameters: Fig. $11 \mathrm{~b}$ and the corresponding results from the previous satellite programs show that the probabilities of $\Delta N_{\mathrm{i}} / N_{\mathrm{i}}>$ $1 \%$ associated with the local time intervals [18:00-21:00 LT) and [03:00-06:00 UT) are at least comparable, if not roughly equal. In sharp contrast with this observation, Fig. $8 \mathrm{~b}$ and Table 2 indicate that the probabilities of $\Delta N_{\mathrm{i}}$ associated with the former local time interval are much greater than the latter, consistently with scintillation observations reported by Basu et al. (1988).

It is particularly important to remark that the C/NOFS satellite is capable of probing irregularities over the relatively wide altitude range [400-800 km) in the topside ionosphere. Figure 12 shows the detrended probability distribution of $\Delta N_{\mathrm{i}} / N_{\mathrm{i}}>1 \%$ recorded during 2012 as a function of longitude sectors and $100 \mathrm{~km}$ altitude intervals. A slow decrease of these probabilities with altitude is observed for most longitude sectors (the maximum ratio between the extreme probability values for each longitude sector is 1.75). There are a few exceptions from this general trend, which show how strongly the normalizing factor $N_{\mathrm{i}}$ can influence the probability distribution of $\Delta N_{\mathrm{i}} / N_{\mathrm{i}}>1 \%$. Namely, note the increases of the probabilities associated with the South American West and the Asian sectors between the lowest two and three altitude intervals, respectively. Also note that the probability of $\Delta N_{\mathrm{i}} / N_{\mathrm{i}}>1 \%$ corresponding to the Indian longitude sector remains virtually constant in the three lowest altitude intervals. However, Fig. 7b and Table 1 show fast decreases (by factors greater than ten) of the probability distribution of $\Delta N_{\mathrm{i}}$ with the altitude interval. No previous satellite program provided similar results from this altitude range.

\section{Conclusions}

Section 4 established that the results from the analysis of the dependences on several geophysical parameters of the detrended probability distribution of $\Delta N_{\mathrm{i}} / N_{\mathrm{i}}>1 \%$ using C/NOFS PLP in situ data were in good agreement with the corresponding ones from previous satellite programs. It also established that the dependences of the detrended probability distributions of $\Delta N_{\mathrm{i}}$ on these parameters, directly relevant to scintillation-oriented studies, as discussed in Sect. 2, cannot be accurately inferred from those of $\Delta N_{\mathrm{i}} / N_{\mathrm{i}}$. To the authors' knowledge, statistical studies of $\Delta N_{\mathrm{i}}$ using in situ data from a wide altitude range in the equatorial topside ionospheric $\mathrm{F}$ region have not been previously reported. Thus, only the main results from Sect. 3 will be summarized in the next paragraphs.

It has been observed that the probabilities of the irregularities detected in the South American East sector remain the

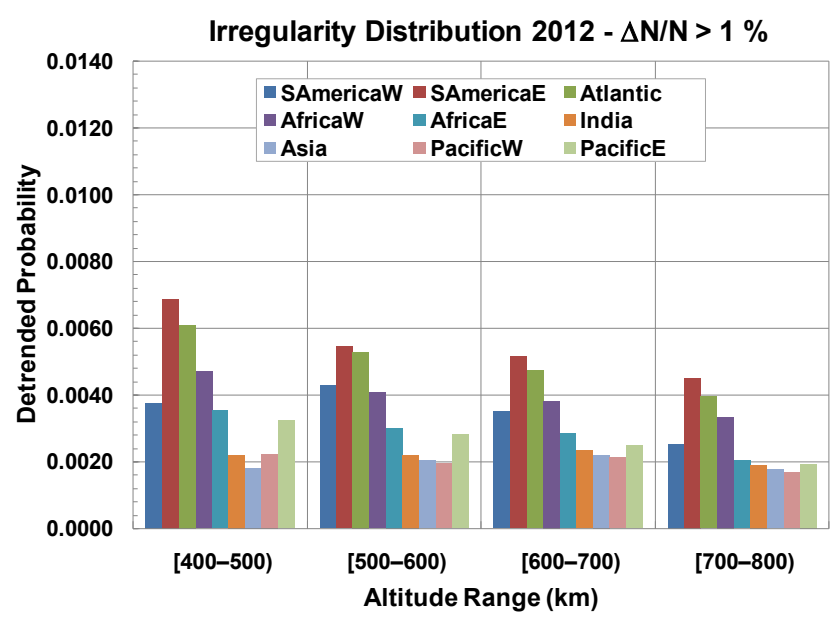

Figure 12. Detrended probability distributions of $\Delta N_{\mathrm{i}} / N_{\mathrm{i}}>1 \%$ as functions of altitude ranges and longitude sectors for 2012 .

largest ones for most combinations of geophysical parameters.

The irregularities with $\log \left(\Delta N_{\mathrm{i}}\right)>3.5$ correspond to $0.72 \%$ of the total number of samples obtained during the evening hours of the solar-minimum year 2008-2009 in the altitude interval $[400-800 \mathrm{~km}$ ). These irregularities are very rare above $500 \mathrm{~km}$, with the exception of those in the South American West and East sectors, which can reach the altitude of $600 \mathrm{~km}$. The probabilities of these irregularities are maximum in the [21:00-24:00 LT) interval for some longitudinal sectors, are extremely rare after 03:00 LT, and peak in the $\left[-5\right.$ to $\left.+5^{\circ}\right]$ magnetic latitude interval for all longitude sectors. This observation is consistent with the field-alignment of irregularities and their concentration in the lowest altitude range. The probability distributions associated with the South American East and Pacific sectors are asymmetric and symmetric around the magnetic equator, respectively.

The irregularities with $\log \left(\Delta N_{\mathrm{i}}\right)>3.5$ correspond to $11.40 \%$ of the total number of samples obtained during the year 2012 under the restrictions of the previous paragraph. The probabilities of these irregularities peak in the lowest altitude range and earliest local time interval. They reach the highest altitude range and are present in the latest local time interval with nonnegligible probabilities for all the sectors. It is particularly interesting to note that, although the probability associated with the Pacific East sector is greater than those of the South American West, Atlantic, and African West sectors for the lowest altitude range, this order is reversed for higher altitudes.

Exponential trend lines depending on altitude and local time have been least-square fitted to the respective set of probabilities corresponding to the irregularities with $\log \left(\Delta N_{\mathrm{i}}\right)>3.5$ for the year 2012 in each longitude sector. The high values obtained for the coefficient of determination $R^{2}$ indicate that, for each longitude sector, an exponential 
trend line provides an acceptable description of the rates at which the probabilities decrease with altitude and local time. However, it should be stressed that the last observation does not imply that the selected exponential form provides an optimum fit to the data or a physical model for the dependence between the parameters under study.

The probabilities of irregularities with $\log \left(\Delta N_{\mathrm{i}}\right)>3.5$ for the year 2012 are more evenly distributed among the magnetic latitude intervals than the ones obtained for the year 2008-2009. The probabilities of the 2012 irregularities in the South American East sector decrease in the following order of magnetic latitude intervals: $\left[-10\right.$ to $\left.-20^{\circ}\right],\left[-5\right.$ to $\left.+5^{\circ}\right]$, and $\left[+10\right.$ to $+20^{\circ}$ ], but this order is reversed for the Pacific sector.

The strong irregularities with $\log \left(\Delta N_{\mathrm{i}}\right)>4.5$ correspond to $0.46 \%$ of the total number of samples obtained during the evening hours of the year 2012 in the altitude interval [400$800 \mathrm{~km}$ ). Their probabilities peak in the lowest altitude range [400-500 km) for all longitude sectors. Only those detected in the South American West sector reach the altitude range [600-700 km) with a nonnegligible probability. The same order reversal with altitude among the probabilities associated with the Pacific East, South American West, and African West sectors also applies to the strong irregularities. The strong irregularities detected in all sectors are also very rare after 24:00 LT. The probabilities corresponding to the South American East, Atlantic, Indian, and Asian sectors have their maximum values in the earliest interval and the other ones in the immediately following local time interval. For fixed longitude sectors, the respective distributions of probabilities of irregularities with $\log \left(\Delta N_{\mathrm{i}}\right)>3.5$ and $\log \left(\Delta N_{\mathrm{i}}\right)>4.5$ display essentially similar dependences on the magnetic latitude interval.

Finally, it should be noted that, for most longitude sectors, the probabilities reach their minimum values during the June solstice. There are exceptions to this general behavior: the probabilities of the irregularities in the Pacific West and East sectors, as well as those of the strong irregularities with $\log \left(\Delta N_{\mathrm{i}}\right)>4.5$ detected during 2012 in the African West, African East, and Asian sectors, reach their relative minimum during the December solstice. However, the probabilities reach their maximum values during the March equinox for a predominant list of combination of longitude sector, year under study, and threshold. For other combinations of the same parameters, the maximum probability can also be reached in all the other seasons.

Acknowledgements. E. Costa was supported by AFOSR award no. FA9550-12-1-0031 during the present work. The authors thank the reviewers for the insightful and constructive comments, which helped them in the development of a better paper.

Topical Editor F. Rodrigues thanks K. Groves and four anonymous referees for their help in evaluating this paper.

\section{References}

Abdu, M. A., Bittencourt, J. A., and Batista, I. S.: Magnetic declination control of the equatorial $\mathrm{F}$ region dynamo electric field development and spread F, J. Geophys. Res., 86, 11443-11446, doi:10.1029/JA086iA13p11443, 1981.

Basu, S., MacKenzie, E., and Basu, Su.: Ionospheric constraints on $\mathrm{VHF} / \mathrm{UHF}$ communications links during solar maximum and minimum periods, Radio Sci., 23, 363-378, doi:10.1029/RS023i003p00363, 1988.

Basu, Su., Basu, S., and Khan, B. K.: Model of equatorial scintillation from in-situ measurements, Radio Sci., 11, 821-832, 1976.

Burke, W. J., Huang, C. Y., Gentile, L. C., and Bauer, L.: Seasonallongitudinal variability of equatorial plasma bubbles, Ann. Geophys., 22, 3089-3098, doi:10.5194/angeo-22-3089-2004, 2004a.

Burke, W. J., Gentile, L. C., Huang, C. Y., Valladares, C. E., and Su, S.-Y.: Longitudinal variability of equatorial plasma bubbles observed by DMSP and ROCSAT, J. Geophys. Res., 109, A12301, doi:10.1029/2004JA010583, 2004b.

Carrasco, A. J. and Batista, I. S.: Estimation of the initial amplitude of plasma bubble seed perturbation from ionograms, Radio Sci., 47, RS2008, doi:10.1029/2011RS004862, 2012.

Costa, E. and Basu, S.: A radio wave scattering algorithm and irregularity model for scintillation predictions, Radio Sci., 37, 18/118/13, doi:10.1029/2001RS002498, 2002.

Costa, E. and Kelley, M. C.: Ionospheric scintillation calculations based on in-situ irregularity spectra, Radio Sci., 12, 797-809, 1977.

Dao, E., Kelley, M. C., Roddy, P. A., Retterer, J. M., Ballenthin, J. O., de La Beaujardiere, O., and Su, Y.-J.: Longitudinal and seasonal dependence of nighttime equatorial plasma density irregularities during solar minimum detected on the C/NOFS satellite: Geophys. Res. Lett., 38, L10104, doi:10.1029/2011GL047046, 2011.

de La Beaujardière, O. and the C/NOFS Science Definition Team: C/NOFS: a mission to forecast scintillation, J. Atmos. Sol.-Terr. Phys., 66, 1573-1591, doi:10.1016/j.jastp.2004.07.030, 2004.

Fortes, J. M. P., Sampaio-Neto, R., and Maldonado, J. E. A.: An analytical method for assessing interference in interference environments involving NGSO satellite networks, Int. J. Satell. Commun. Netw., 17, 399-419, doi:10.1002/(SICI)10991247(199911/12)17:6<399::AID-SAT647>3.0.CO;2-A, 1999.

Fritts, D. C. and Alexander, M. J.: Gravity wave dynamics and effects in the middle atmosphere, Rev. Geophys., 41, 1003, doi:10.1029/2001RG000106, 2003.

Groves, K. M., Basu, S., Weber, E. J., Smitham, M., Kuenzler, H., Valladares, C. E., Sheehan, R., MacKenzie, E., Secan, J. A., Ning, P., McNeill, W. J., Moonan, D. W., and Kendra, M. J.: Equatorial scintillation and systems support, Radio Sci., 32, 2047-2064, doi:10.1029/97RS00836, 1997.

Haykin, S.: Communication Systems, 2nd Edn., Wiley, New York, 1983.

Huang, C.-S., de La Beaujardiere, O., Roddy, P. A., Hunton, D. E., Pfaff Jr., R. F., Valladares, C. E., and Ballenthin, J. O.: Evolution of equatorial ionospheric plasma bubbles and formation of broad plasma depletions measured by the C/NOFS satellite during deep solar minimum, J. Geophys. Res., 116, A03309, doi:10.1029/2010JA015982, 2011.

Huang, C. Y., Burke, W. J., Machuzak, J. S., Gentile, L. C., and Sultan, P. J.: DMSP observations of equatorial plasma bubbles 
in the topside ionosphere near solar maximum, J. Geophys. Res., 106, 8131-8142, 2001.

Huang, C. Y., Burke, W. J., Machuzak, J. S., Gentile, L. C., and Sultan, P. J.: Equatorial plasma bubbles observed by DMSP satellites during a full solar cycle: Toward a global climatology, J. Geophys. Res., 107, 1434, doi:10.1029/2002JA009452, 2002.

International Association of Geomagnetism and Aeronomy and Working Group V-MOD participating members (Finlay, C. C., Maus, S., Beggan, C. D., Bondar, T. N., Chambodut, A., Chernova, T. A., Chulliat, A., Golovkov, V. P., Hamilton, B., Hamoudi, M., Holme, R., Hulot, G., Kuang, W., Langlais, B., Lesur, V., Lowes, F. J., Lühr, H., Macmillan, S., Mandea, M., McLean, S., Manoj, C., Menvielle, M., Michaelis, I., Olsen, N., Rauberg, J., Rother, M., Sabaka, T. J., Tangborn, A., TøffnerClausen, L., Thébault, E., Thomson, A. W. P., Wardinski, I., Wei, Z., and Zvereva, T. I.): International Geomagnetic Reference Field: the eleventh generation, Geophys. J. Int., 183, 12161230, doi:10.1111/j.1365-246X.2010.04804.x, 2010.

International Telecommunication Union (ITU-R): Analytical method for determining the statistics of interference between non-geostationary-satellite orbit fixed-satellite service systems and other non-geostationary-satellite orbit fixed-satellite service systems or geostationary-satellite orbit fixed-satellite service networks, Recommendation ITU-R S.1529, Geneva, 2001.

Kil, Y. and Heelis, R. A.: Global distribution of density irregularities in the equatorial ionosphere, J. Geophys. Res., 103, 407-417, 1998.

McClure, J. P., Singh, S., Bamgboye, D. K.,Johnson, F. S., and Kil, Y.: Occurrence of equatorial $\mathrm{F}$ region irregularities: Evidence for tropospheric seeding, J. Geophys. Res., 103, 29119-29135, 1998.

Nishioka, M., Basu, Su., Basu, S., Valladares, C. E., Sheehan, R. E., Roddy, P. A., and Groves, K. M.: C/NOFS satellite observations of equatorial ionospheric plasma structures supported by multiple ground-based diagnostics in October 2008, J. Geophys. Res., 116, A10323, doi:10.1029/2011JA016446, 2011.

Papoulis, A.: Probability, Random Variables, and Stochastic Processes, 3rd Edn., McGraw-Hill, New York, 1991.

Retterer, J. M.: Forecasting low-latitude radio scintillation with 3D ionospheric plume models: 1. Plume model, J. Geophys. Res., 115, A03306, doi:10.1029/2008JA013839, 2010.
Rino, C. L.: A power law phase screen model for ionospheric scintillation: 1. weak scatter, Radio Sci., 14, 1135-1145, 1979.

Roddy, P. A., Hunton, D. E., Ballenthin, J. O., and Groves, K. M.: Correlation of in situ measurements of plasma irregularities with ground-based scintillation observations, J. Geophys. Res., 115, A06303, doi:10.1029/2010JA015288, 2010.

Rufenach, C. L.: Ionospheric scintillation by a random phase screen: spectral approach, Radio Sci., 10, 155-165, 1975.

Su, S.-Y., Liu, C. H., Ho, H. H., and Chao, C. K.: Distribution characteristics of topside ionospheric density irregularities: Equatorial versus midlatitude regions, J. Geophys. Res., 111, A06305, doi:10.1029/2005JA011330, 2006.

Su, Y.-J., Retterer, J. M., de La Beaujardiere, O., Burke, W. J., Roddy, P. A., Pfaff Jr., R. F., Wilson, G. R., and Hunton, D. E.: Assimilative modeling of equatorial plasma depletions observed by C/NOFS, Geophys. Res. Lett., 36, L00C02, doi:10.1029/2009GL038946, 2009.

Su, Y.-J., Retterer, J. M., Pfaff Jr., R. F., Roddy, P. A., de La Beaujardière, O., and Ballenthin, J. O.: Assimilative modeling of observed postmidnight equatorial plasma depletions in June 2008, J. Geophys. Res., 116, A09318, doi:10.1029/2011JA016772, 2011.

Takahashi, H., Taylor, M. J., Pautet, P.-D., Medeiros, A. F., Gobbi, D., Wrasse, C. M., Fechine, J., Abdu, M. A., Batista, I. S., Paula, E., Sobral, J. H. A., Arruda, D., Vadas, S. L., Sabbas, F. S., and Fritts, D. C.: Simultaneous observation of ionospheric plasma bubbles and mesospheric gravity waves during the SpreadFEx Campaign, Ann. Geophys., 27, 1477-1487, doi:10.5194/angeo27-1477-2009, 2009.

Tsunoda, R. T.: Control of the seasonal and longitudinal occurrence of equatorial scintillations by the longitudinal gradient in integrated E region Pedersen conductivity, J. Geophys. Res., 90, 447-456, doi:10.1029/JA090iA01p00447, 1985.

Yeh, K. C. and Liu, C. H.: Radio wave scintillations in the ionosphere, IEEE Proc., 70, 324-360, doi:10.1109/PROC.1982.12313, 1982. 